\title{
The role of spurions in Higgs-less electroweak effective theories
}

\author{
Johannes Hirn ${ }^{1,2, a}$ and Jan Stern ${ }^{2, b}$ \\ 1 Groupe Physique Théorique ${ }^{\mathrm{c}}$, IPN Orsay, Université Paris-Sud XI, 91406 Orsay, France \\ 2 Institute for Particle Physics Phenomenology, University of Durham, Durham DH1 3LE, UK
}

\begin{abstract}
Inspired by recent developments of moose models, we reconsider low-energy effective theories of Goldstone bosons, gauge fields and chiral fermions applied to low-energy QCD and to Higgs-less electroweak symmetry breaking. Couplings and the corresponding reduction of symmetry are introduced via constraints enforced by a set of non-propagating covariantly constant spurion fields. Relics of the latter are used as small expansion parameters conjointly with the usual low-energy expansion. Certain couplings can only appear at higher orders of the spurion expansion and, consequently, they become naturally suppressed independently of the idea of dimensional deconstruction. At leading order this leads to a set of generalized Weinberg sum rules and to the suppression of non-standard couplings to fermions in Higgs-less EWSB models with the minimal particle content. Within the latter, higher spurion terms allow for a fermion mass matrix with the standard CKM structure and $C P$ violation. In addition, Majorana masses for neutrinos are possible. Examples of non-minimal models are briefly mentioned.
\end{abstract}

\section{Introduction}

In a low-energy effective theory (LEET) 1], the requirement of naturalness 2] plays a central role in defining a systematic low-energy expansion that is finite order by order despite the lack of renormalizability and a bad highenergy behavior. LEET operates with light degrees of freedom, which become massless in a particular limit, due to chiral and/or gauge symmetries. The lagrangian is constructed and renormalized order-by-order in a momentum expansion. Naturalness then amounts to the requirement that, at each order, the lagrangian contains all terms allowed by the symmetries and by the power counting. The simplest example is Chiral Perturbation Theory $(\chi \mathrm{PT})[3$. 4 - the low-energy effective theory of QCD. It merely operates with Goldstone bosons of spontaneously broken chiral symmetry. Since in this case, the high-energy completion of the effective theory (i.e. QCD) is known, the $\mathcal{O}\left(p^{4}\right)$ low-energy constants (LECs) can be put under partial phenomenological control.

In this article, we are particularly concerned with more complicated effective theories which, in addition to Goldstone bosons, also contain gauge fields. For some time this type of LEET was hoped to describe electroweak symmetry breaking (EWSB) resulting from the dynamical Higgs mechanism [5, 6. Especially interesting was the possibility of EWSB with no scalar particles remaining in the spectrum. The question whether a natural and phenomenologically viable LEET of EWSB without scalar relics exists

\footnotetext{
a e-mail: johannes.hirn@durham.ac.uk

b e-mail: stern@ipno.in2p3.fr

c Unité mixte de recherche 8608.
}

is still of interest today as it would represent an alternative to the standard interpretation of electroweak precision tests as constraints on the Higgs mass 7]. Unfortunately, this type of effective theories lacks predictivity at $\mathcal{O}\left(p^{4}\right)$ order (i.e. one loop), since its high-energy completion is not known: the values of the renormalized $\mathcal{O}\left(p^{4}\right)$ LECs can so far only be discussed within particular models such as "rescaled QCD" 8, or the gauged linear sigma model in the heavy-mass limit 9,10,11,12. Beyond those models, it seems premature to conclude that a generic Higgs-less LEET of EWSB is at variance with precision electroweak tests. On the other hand, already at leading $\mathcal{O}\left(p^{2}\right)$ order, not all couplings one may construct in a generic Higgs-less effective theory based on the symmetry arguments alone are actually observed.

i) Tree-level $\mathcal{O}\left(p^{2}\right)$ contributions to the $S$ parameter are in principle possible.

ii) There are non-standard couplings to fermions, which would spoil universality of the left-handed couplings and introduce right-handed couplings of the $W^{ \pm}$. This is unacceptable at leading order and the problem we address is that of suppressing those non-standard couplings naturally.

iii) Last but not least, let us mention the long-standing problem of fermion masses and flavor symmetry breaking. Indeed, within Higgs-less effective theories it has always been difficult to have, at leading order, mass-splittings within a doublet of the same order of magnitude as the masses themselves.

All these problems suggest that something else should be added to the momentum expansion based on the lowenergy symmetry $\mathrm{SU}(2) \times \mathrm{U}(1)_{Y}$ in order to construct natural Higgs-less effective theories of EWSB. 
Johannes Hirn, Jan Stern: The role of spurions in Higgs-less electroweak effective theories

It is interesting to note that a similar problem in principle arises if the low-energy effective theory of QCD is extended beyond the pure $\chi \mathrm{PT}$ framework towards higher energies, incorporating vector and axial vector states. If the latter are included among protected light states of a LEET, they should be treated as weakly coupled gauge particles (although such a scenario need not be realistic for finite- $N_{c}$ QCD, we are going to use it as a simple theoretical laboratory 13). If in the corresponding LEET all terms allowed by the chiral symmetry were kept 14 . 15], the Weinberg sum rules (WSRs) [16] would be lost. In QCD, the latter do not follow from chiral symmetry alone, but hinge strongly on short-distance properties of the theory. Within LEET this leads once more to the problem mentioned in connection with EWSB: how to naturally suppress unwanted terms in order to recover the two Weinberg sum rules and obtain finite radiative masses for pseudo-Goldstone bosons (PGBs).

Our starting point is therefore a recent discussion of the two Weinberg sum rules [17, based on moose models 18. The model considered consists in a chain of Goldstone bosons of spontaneously broken chiral symmetries coupled in a particular way to gauge fields. Not all couplings allowed by the symmetries and low-energy power counting are admitted, leading to a better high-energy behavior than one might expect in effective theories exclusively based on the low-energy symmetries. The literature does not relate these properties to a requirement of symmetry. Indeed, the standard line of thinking has been that of dimensional deconstruction [19 20, in which case unwanted terms in the lagrangian are omitted on a physical basis: the requirement of locality along the fifth (discretized) dimension. Only interactions between nearest neighbors along the moose are then allowed. What happens at higher orders is then unclear: such terms will be generated and one can question whether it is a licit procedure to omit them altogether in the first place.

What we would like to demonstrate here is the feasibility and the usefulness of an alternative bottom-up approach, without reference to a five-dimensional theory. Instead we will focus on an approach which is closely linked with the naturalness hypothesis formulated by 't Hooft 2 , that is, relying on the symmetries of the theory. Our formulation will make extensive use of non-propagating fields - called spurions - within the LEET 1,3], in order to keep track of the reduction of symmetry when the gauge couplings are introduced. These spurions and the constraints applied on them serve the purpose of introducing couplings by restricting the configuration space of gauge connections, while at the same time allowing the use of a lagrangian which is invariant under the full symmetry. Spurions have been employed in the past in various contexts: explicit chiral symmetry breaking due to quark masses 113, introduction of electromagnetic couplings in $\chi \mathrm{PT}$ 21 22, radiative masses of PGBs 23, and also linear moose models 24]. In 24, spurions were used to formally count the occurrences of coupling constants in connection with radiative corrections to PGBs masses once the unwanted couplings have been dismissed. At the end, the spurions of reference 24] are set to one. In this paper we go one step further: we consider covariantly constant spurions as small expansion parameters which are a genuine part of the effective theory, allowing for a natural suppression of unwanted couplings between Goldstone bosons and gauge fields. The LEET will be constructed as a simultaneous expansion in powers of momenta, (gauge) couplings and spurions 1,3,4,22: unwanted couplings will be suppressed since they only appear at higher orders of the spurion expansion. The spurion formalism developed in this paper represents a general device to couple $\mathrm{SU}(2) \times \mathrm{SU}(2)$ Goldstone bosons and $\mathrm{SU}(2)$ gauge fields. As such, it applies both to effective theories of QCD and of Higgs-less EWSB. This is the main reason why these apparently distinct subjects are discussed jointly within the same paper. The paper is organized as follows.

In section 2 we introduce real covariantly-constant spurions in the general linear moose model based on $\mathrm{SU}(2)$ groups. We then perform appropriate field redefinitions to study the spectrum of the theory, which consists in a multiplet of Goldstone bosons and a tower of massive vectors.

In section 3 we consider the left-right two-point correlator and show that, at leading order in the spurion expansion, and in the tree-level approximation (cf. the large- $N_{c}$ limit of QCD), it automatically satisfies $K$ generalized Weinberg sum rules, where $K$ is the length of the moose. Higher spurion terms introduce corrections to these WSRs. We give the expression for $L_{10}$, which is related to the $S$ parameter of the electroweak sector.

In section 4 we consider in detail the Higgs-less effective EWSB theory with a minimal particle content: electroweak bosons and fermions. There are no scalar particles below the scale $4 \pi f(f \simeq 250 \mathrm{GeV})$ where the effective theory breaks down. The minimal set of spurions needed to select the reduced symmetry $\mathrm{SU}(2) \times \mathrm{U}(1)_{Y}$ out of a larger $\mathrm{SU}(2)^{4} \times \mathrm{U}(1)_{B-L}$ natural symmetry is discussed. It is shown how a complex spurion selects the correct $\mathrm{U}(1)$ subgroup and describes weak isospin breaking effects. The suppression of all non-standard couplings to fermions is demonstrated at the leading order of the spurion expansion. It is further shown that spurions allow the introduction of fermion mass-matrices with a general CKM structure and $C P$ violation.

In section 5 we describe two non-minimal models of EWSB based on extension of the moose of section 4 The first of these two models contains $W^{\prime}$ and $Z^{\prime}$ excited vectors. Our purpose is to exhibit what the first corrections to the $S, T, U$ parameters might be. Our last model does not contain excited vectors in the low-energy sector, but rather a triplet of PGBs. We illustrate the role of the Weinberg sum rules discussed in section 3 in relation with the radiative masses of these PGBs. This last model provides an example of light scalars which do not play the same role as the standard Higgs boson. Again, we introduce fermions in the model and discuss the consequences of the spurion power counting.

We summarize our findings in section [6] 


\section{Spurions and mooses}

In this section, the open linear moose is studied at lowest order in the momentum expansion and lowest order in the spurion power counting: interactions between Goldstone bosons and gauge fields are introduced via constraints imposed on spurions embodying the principle of naturalness. Indeed, we start with a theory with Goldstone multiplets and gauge fields. When the parameters are sent to appropriate limits, the separate theories do not interact anymore. Consequently, it is sensible to consider an expansion where the parameters are close to these limits: this is both a well-defined expansion from the practical point of view and a meaningful one in the sense that the symmetry is increased when the parameters are set to be equal to the aforementioned limits. This in turn makes it logically plausible that an underlying theory produces this set of values for the parameters without fine-tuning because the symmetry preserves this situation from obtaining large corrections. We want to build a perturbative expansion around the limit where the Goldstone bosons do not interact with the gauge fields: the moose is then disconnected.

In order to disentangle the disconnected limit from the case when the gauge coupling constants are taken to zero, we are compelled to introduce a set of additional parameters, which can be taken to zero independently. These parameters will in fact derive from spurion fields, which render the theory invariant under a larger symmetry group: the group of transformations where the chiral transformations on the Goldstone fields are independent of the gauge transformations. From this we may deduce how the spurions will have to transform. We find that the connections corresponding to the chiral transformations on the Goldstone fields and the gauge connections become identified, and that the spurions reduce to constants, which will become our small expansion parameters.

In this section, we focus on spurions satisfying, in addition to the constraint of covariant constancy, a reality condition. This will allow for each SU $(2)^{2}$ symmetry under which the spurion transforms to be restricted to $\mathrm{SU}(2)$. Later on in sections 4 and 5 we will add complex spurions in order to reduce the symmetry further to $U(1)$.

\subsection{Goldstone bosons and Gauge fields}

We consider $K+1$ independent non-linear sigma models, each describing the spontaneous breakdown SU $(2) \times$ $\mathrm{SU}(2) \longrightarrow \mathrm{SU}(2)$. In addition, we consider $K$ independent Yang-Mills theories with gauge group SU(2). We will describe the general case, leaving $K$ as a free parameter, but will pick the particular case with $K=2$ for definiteness in order to draw diagrams in this section, such as Fig. 2.1. This diagram describes the symmetries of the model: for this particular case, we really have five completely independent theories at this stage. The three non-linear sigma models are depicted as oriented links between two squares representing the transformation operating on the Goldstone multiplets. For instance the leftmost item in the figure represents the fact that the unitary matrix $\Sigma_{0,1}$ transforms as

$$
\Sigma_{0,1} \longmapsto L_{0} \Sigma_{0,1} R_{1}^{\dagger}
$$

with $\left(L_{0}, R_{1}\right) \in \mathrm{SU}(2) \times \mathrm{SU}(2)$. The two gauge groups are represented by circles, and in fact the circle labeled $G_{1}$ means that the gauge transformation on the hermitian gauge field $G_{1 \mu}$ is given by

$$
G_{1 \mu} \longmapsto G_{1} G_{1 \mu} G_{1}^{\dagger}+\frac{\mathrm{i}}{g_{1}} G_{1} \partial_{\mu} G_{1}^{\dagger},
$$

with $G_{1} \in \mathrm{SU}(2)$. The positioning and naming of the various elements in the diagram is irrelevant at this stage, although the different pieces will be connected later on to form a chain.

Coming back to the general case, we point out that the model we have just introduced possesses the symmetry

$$
\begin{aligned}
S_{\text {natural }, K} & =\prod_{l=1}^{K} \mathrm{SU}(2)_{G_{l}} \\
& \times \prod_{k=0}^{K} \mathrm{SU}(2)_{L_{k}} \times \mathrm{SU}(2)_{R_{k+1}}
\end{aligned}
$$

under which the fields transform as

$$
\begin{aligned}
& \Sigma_{k, k+1} \longmapsto L_{k} \Sigma_{k, k+1} R_{k+1}^{\dagger}, \text { for } k=0, \cdots, K, \\
& G_{k \mu} \longmapsto G_{k} G_{k \mu} G_{k}^{\dagger}+\frac{\mathrm{i}}{g_{k}} G_{k} \partial_{\mu} G_{k}^{\dagger}, \\
& \quad \text { for } k=1, \cdots, K,
\end{aligned}
$$

where we have introduced the SU (2) transformations $L_{k}$, $R_{k}, G_{k}$. The standard practice consists in considering $l o-$ cal chiral transformations $L_{k}, R_{k}$, in order to define the generating functional for the Noether currents of the corresponding symmetries 3,4]. We will rely heavily on this in the following and we therefore introduce the following covariant derivatives

$$
\begin{gathered}
D_{\mu} \Sigma_{k, k+1}=\partial_{\mu} \Sigma_{k, k+1}-\mathrm{i} L_{k \mu} \Sigma_{k, k+1}+\mathrm{i} \Sigma_{k, k+1} R_{k+1 \mu}, \\
\text { for } k=0, \cdots, K
\end{gathered}
$$

where the $L_{k \mu}$ and $R_{k \mu}$ are sources.

Now, we want to build a LEET characterized by an expansion in powers of momenta, the momentum scale being set by the momenta of light external particles. Therefore, we assume slowly varying external sources and consider an expansion in powers of derivatives. In order to write down the most general lagrangian consistent with the symmetry $S_{\text {natural,K}}$ of the problem, we note that if one performs the following transformations on the sources under the full group $S_{\text {natural }, K}$

$$
\begin{aligned}
& L_{k \mu} \longmapsto L_{k} L_{k \mu} L_{k}^{\dagger}+\mathrm{i} L_{k} \partial_{\mu} L_{k}^{\dagger}, \\
& R_{k \mu} \longmapsto R_{k} R_{k \mu} R_{k}^{\dagger}+\mathrm{i} R_{k} \partial_{\mu} R_{k}^{\dagger},
\end{aligned}
$$

then the effective lagrangian must be invariant in order to reproduce the Ward identities of the theory [25], up to possible fermion anomalies [26, which only impact the nextto-leading order in the momentum expansion 3 . We use 

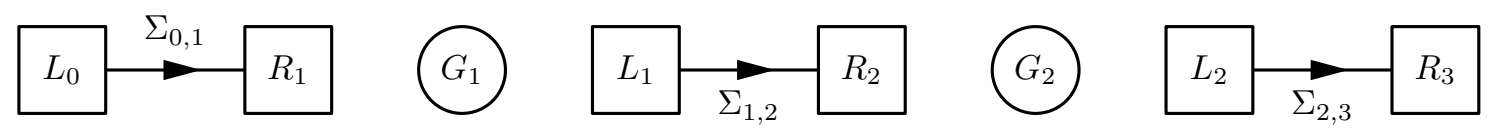

Fig. 2.1. Three non-linear sigma models and two Yang-Mills theories.

the appropriate power counting for covariant derivatives and gauge fields 22,27

$$
\begin{aligned}
g_{k} & =\mathcal{O}\left(p^{1}\right), \\
G_{k \mu} & =\mathcal{O}\left(p^{0}\right), \\
L_{k \mu}, R_{k \mu} & =\mathcal{O}\left(p^{1}\right),
\end{aligned}
$$

defined to have the connections and derivatives counted on the same footing, in order to obtain a covariant expansion order by order, and to have the kinetic terms for the dynamical gauge fields appear at the same order as that for the Goldstone bosons. Alternatively, one may consider the normalization of states for this last step. With these rules we get at lowest order in the expansion in powers of momenta, that is to say at $\mathcal{O}\left(p^{2}\right)$, the following lagrangian

$$
\begin{aligned}
\mathcal{L} & =\frac{1}{4} \sum_{k=0}^{K} f_{k}^{2}\left\langle D_{\mu} \Sigma_{k, k+1} D^{\mu} \Sigma_{k, k+1}^{\dagger}\right\rangle \\
& -\frac{1}{2} \sum_{k=1}^{K}\left\langle G_{k \mu \nu} G_{k}^{\mu \nu}\right\rangle .
\end{aligned}
$$

In the above, the usual definition for the field-strength applies

$$
\begin{gathered}
G_{k \mu \nu}=\partial_{\mu} G_{k \mu}-\partial_{\nu} G_{k \mu}-\mathrm{i} g_{k}\left[G_{k \mu}, G_{k \nu}\right], \\
\quad \text { for } k=1, \ldots, K
\end{gathered}
$$

and the symbol $\langle\cdots\rangle$ denotes the trace of the two-by-two matrices.

\subsection{Couplings along the chain}

We now wish to introduce couplings between the Goldstone multiplets via interactions with gauge fields. However, the lagrangian (2.12) does not describe interactions between the disconnected elements of Fig. 2.1 nor does it suggest any line-ordering either, except for the relative positions we have chosen for the purpose of the diagram. To remedy this, we now introduce couplings between the independent theories, not by adding new interaction terms, but by restricting fields in the lagrangian through constraints ${ }^{1}$.

With this objective in mind, we now consider spurions, which we introduce as non-propagating fields satisfying some constraints. These constraints will restrict the allowed space of gauge configurations. In other words, they imply identifications between various symmetry transformations operating on the model, while enabling us to keep the full invariance group $S_{\text {natural, } K}$ of the original theory.

\footnotetext{
1 This is quite standard: for instance the non-linear sigma model itself introduces interactions in this manner.
}

\subsubsection{Real spurions}

In this section, we introduce spurions as two-by-two matrixvalued fields $X_{k}, Y_{k}$ for $k=1, \cdots, K$, subject to a reality condition. The constraint of covariant constancy is then imposed on them. We show that one can find a gauge in which the spurions reduce to real constants times the identity matrix

$$
\begin{aligned}
\left.X_{k}\right|_{\text {const. }} & =\xi_{k} \mathbb{1}_{2 \times 2}, \\
\left.Y_{k}\right|_{\text {const. }} & =\eta_{k} \mathbb{1}_{2 \times 2} .
\end{aligned}
$$

The constants $\xi_{k}, \eta_{k}$ are assumed to be small, and taken as expansion parameters. The constraints are solved in this gauge to yield

$$
\begin{aligned}
& \left.R_{k \mu}^{a}\right|_{\text {const. }}=g_{k} G_{k \mu}^{a}, \text { for } a=1,2,3, \\
& \left.L_{k \mu}^{a}\right|_{\text {const. }}=g_{k} G_{k \mu}^{a}, \text { for } a=1,2,3,
\end{aligned}
$$

implying that the connections are identified, thereby introducing the couplings of Goldstone fields to gauge fields. The $G_{k}$ transformation is unconstrained.

We now proceed to describe the spurions and the constraints, before solving these: in the setting of section 2.1 we introduce the matrices $X_{k}, Y_{k}$ with the assumed transformation properties

$$
\begin{aligned}
& X_{k} \longmapsto R_{k} X_{k} G_{k}^{\dagger}, \text { for } k=1, \ldots, K, \\
& Y_{k} \longmapsto G_{k} Y_{k} L_{k}^{\dagger}, \text { for } k=1, \ldots, K,
\end{aligned}
$$

as depicted in Fig. 2.2 (compare figure 2.1) . These spurions serve the purpose of relating the gauge transformations with the chiral transformations operating on the Goldstone multiplets. In relation with the hypothesis of naturalness and the limit in which the Goldstone bosons and the gauge fields do not interact with each other, we will consider the case where the entries in these spurions are functions with a small modulus, and therefore consider

$$
X_{k}, Y_{k}=\mathcal{O}(\epsilon), \text { for } k=1, \ldots, K,
$$

where $\epsilon$ is by assumption a small parameter. We have assumed the simplest situation here, considering all spurions to be of the same order of magnitude. This is however not necessarily the case and at this point we have no way of deciding what the appropriate counting is. We will therefore continue with this simple assumption.

In order to introduce the gauge couplings to the Goldstone bosons via the identification of connections, we now demand that the spurions satisfy a constraint. Since we want the spurions to reduce to constants once the constraints are enforced while maintaining a covariant formulation and at the same time identifying the connections, 


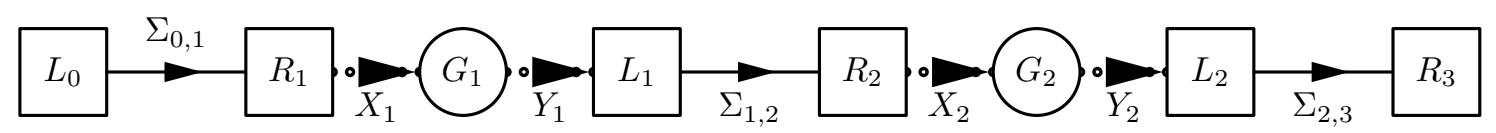

Fig. 2.2. Introduction of spurions in the model of section 2.1

it seems natural to impose the condition of covariant constancy $^{2}$

$$
\begin{aligned}
D_{\mu} X_{k} & =0, \text { for } k=1, \ldots, K, \\
D_{\mu} Y_{k} & =0, \text { for } k=1, \ldots, K .
\end{aligned}
$$

From (2.18) and (2.19) we deduce that the covariant derivatives in the above equations are given by

$$
\begin{aligned}
D_{\mu} X_{k} & =\partial_{\mu} X_{k}-\mathrm{i} R_{k \mu} X_{k}+\mathrm{i} g_{k} X_{k} G_{k \mu}, \\
D_{\mu} Y_{k} & =\partial_{\mu} Y_{k}-\mathrm{i} g_{k} G_{k \mu} Y_{k}+\mathrm{i} Y_{k} L_{k \mu} .
\end{aligned}
$$

Notice that the constraints (2.21) and (2.22) restrict both the gauge connections contained in the covariant derivatives and also the non-propagating fields. Now, the nature of the spurion fields itself impacts the way the full symmetry is reduced: we consider in this section the simplest case, imposing the following reality condition on the spurions

$$
\begin{aligned}
& X_{k}^{c}=X_{k}, \text { for } k=1, \ldots, K, \\
& Y_{k}^{c}=Y_{k}, \text { for } k=1, \ldots, K,
\end{aligned}
$$

where we have defined the conjugate $X^{c}$ of any two-by-two matrix $X$ as

$$
X^{c}=\tau^{2} X^{*} \tau^{2}
$$

Note that the reality conditions (2.25) and (2.26) are compatible with the transformations (2.18) and (2.19). This fact, as well as the reality condition itself, is specific to the group SU (2). The reality conditions (2.25) and (2.26) are in fact equivalent to the statement that we may write

$$
\begin{aligned}
X_{k} & =\xi_{k} U_{k}, \text { for } k=1, \ldots, K, \\
Y_{k} & =\eta_{k} V_{k}, \text { for } k=1, \ldots, K,
\end{aligned}
$$

where $\xi_{k}, \eta_{k}$ are real functions and $U_{k}$ and $V_{k}$ are SU (2) matrices. From (2.20) we see that the small parameter is the magnitude of the functions $\xi_{k}, \eta_{k}$

$$
\xi_{k}, \eta_{k}=\mathcal{O}(\epsilon), \text { for } k=1, \ldots, K
$$

In order to demonstrate our claims (2.14 2.17), we perform an SU $(2)_{R_{k}}$ transformation with the following parameter

$$
R_{k}=U_{k}^{\dagger}
$$

Identifying the components in the constraint (2.21), one then obtains that $X_{k}$ is written as in (2.14), with

$$
\partial_{\mu} \xi_{k}=0
$$

\footnotetext{
2 Note that such a constraint could be enforced using Lagrange multipliers. For this simple application, we find it more convenient to impose it by hand.
}

as well as the result announced in (2.16). Following the same steps for $Y_{k}$, with the $\mathrm{SU}(2)_{L_{k}}$ transformation given by

$$
L_{k}=V_{k}
$$

to write $Y_{k}$ in the form of (2.15), we find that the constraint results in

$$
\partial_{\mu} \eta_{k}=0
$$

together with 2.17). Thus, the constraints can be solved easily in the gauge reached by the transformations (2.31) and (2.33), where they imply the identification of the connections with the gauge fields.

Note that we did not have to use the gauge transformation $G_{k}$ corresponding to the dynamical gauge field. This $\mathrm{SU}(2)$ gauge field by itself is therefore free of any constraints. On the other hand, we have performed $R_{k}$ and $L_{k}$ transformations, and the corresponding parameters are no more independent. Hence, we see that the real spurions select in the original $S_{\text {natural }, K}$ group the following subgroup which we denote by $S_{\text {reduced, } K}$

$$
\begin{aligned}
S_{\text {reduced }, K} & =\mathrm{SU}(2)_{L_{0}} \times \mathrm{SU}(2)_{R_{K+1}} \\
& \times \prod_{k=1}^{K} \mathrm{SU}(2)_{R_{k}+G_{k}+L_{k}} .
\end{aligned}
$$

A subgroup of this,

$$
S_{\text {dynamical }, K}=\prod_{k=1}^{K} \mathrm{SU}(2)_{R_{k}+G_{k}+L_{k}},
$$

corresponds to dynamical gauge fields which propagate: this is the subgroup which is 'gauged'. $S_{\text {reduced, } K}$ coincides with the invariance group of the solution (2.14 2.17) to the constraints (2.21) and (2.22) . Defining $S_{\text {natural, } K \text { (2.3) as }}$ the maximal symmetry of a given set of uncoupled Goldstone bosons and Yang Mills fields (c.f. Fig. 2.1]), the list of spurions needed to operate the reduction of $S_{\text {natural, } K}$ to $S_{\text {reduced,K }}$ appears to be fixed essentially uniquely.

For the moment, we have not introduced any spurions transforming under either $L_{0}$ or $R_{K+1}$ and an additional dynamical gauge group. We will consider this in sections 4 and 5 and these additional gauge groups will then be identified with the weak gauge groups. To achieve the proper gauge group reduction, we will be interested in generic spurions for which the reality condition is dropped. This will leave us with the U(1) subgroup of SU (2), but for this section and section 3 , we limit ourselves to cases were the symmetry groups at the end of the moose are not dynamical. 


\subsubsection{Leading-order lagrangian}

Imposing the constraints can be diagrammatically represented by the modification of Fig. 2.2 into a new reduced - notation describing the same model, as shown in Fig. 2.3 This representation of the moose is closer to the standard one found in the literature 19.17): it describes the moose after application of the constraints, which we will call the 'constrained' moose. Note that some information is lost with this diagram as there is no trace of the spurions, which will nonetheless play a prominent role in the sequel.

We also introduce a new notation for the covariant derivative acting on the Goldstone multiplets when the solution to the constraints in the standard gauge as defined in section 2.2.1 is injected, for $k=1, \cdots, K-1$

$$
\begin{aligned}
\nabla_{\mu} \Sigma_{k, k+1} & =\left.D_{\mu} \Sigma_{k, k+1}\right|_{\text {const. }} \\
& =\partial_{\mu} \Sigma_{k, k+1}-\mathrm{i} g_{k} G_{k \mu} \Sigma_{k, k+1} \\
& +\mathrm{i} g_{k+1} \Sigma_{k, k+1} G_{k+1 \mu},
\end{aligned}
$$

and for the ends of the moose

$$
\begin{aligned}
\nabla_{\mu} \Sigma_{0,1} & =\left.D_{\mu} \Sigma_{0,1}\right|_{\text {const. }} \\
& =\partial_{\mu} \Sigma_{0,1}-\mathrm{i} L_{0 \mu} \Sigma_{0,1} \\
& +\mathrm{i} g_{1} \Sigma_{0,1} G_{1 \mu} \\
\nabla_{\mu} \Sigma_{K, K+1} & =\left.D_{\mu} \Sigma_{K, K+1}\right|_{\text {const. }} \\
& =\partial_{\mu} \Sigma_{K, K+1}-\mathrm{i} g_{K} G_{K \mu} \Sigma_{K, K+1} \\
& +\mathrm{i} \Sigma_{K, K+1} R_{K+1 \mu} .
\end{aligned}
$$

Now, we may write the leading-order lagrangian, that is the $\mathcal{O}\left(p^{2} \epsilon^{0}\right)$ lagrangian. In fact, all the terms of this order are already collected in (2.12). This $\mathcal{O}\left(p^{2} \epsilon^{0}\right)$ lagrangian, in which the spurions do not appear explicitly, is reproduced here

$$
\begin{aligned}
\mathcal{L}^{(2,0)} & =\frac{1}{4} \sum_{k=0}^{K} f_{k}^{2}\left\langle D_{\mu} \Sigma_{k, k+1} D^{\mu} \Sigma_{k, k+1}^{\dagger}\right\rangle \\
& -\frac{1}{2} \sum_{k=1}^{K}\left\langle G_{k \mu \nu} G_{k}^{\mu \nu}\right\rangle
\end{aligned}
$$

The point of this spurion formalism is the following: before we impose the constraints (2.21) and (2.22), the Goldstone bosons do not know about the gauge fields. It is only upon injection of the solution to these constraints that we find interactions between the Goldstone bosons and the gauge fields. With the help of the definitions (2.37 2.39) for the $\nabla_{\mu}$ covariant derivatives, we obtain the following 'constrained' $\mathcal{O}\left(p^{2} \epsilon^{0}\right)$ lagrangian

$$
\begin{aligned}
\left.\mathcal{L}^{(2,0)}\right|_{\text {const. }} & =\frac{1}{4} \sum_{k=0}^{K} f_{k}^{2}\left\langle\nabla_{\mu} \Sigma_{k, k+1} \nabla^{\mu} \Sigma_{k, k+1}^{\dagger}\right\rangle \\
& -\frac{1}{2} \sum_{k=1}^{K}\left\langle G_{k \mu \nu} G_{k}^{\mu \nu}\right\rangle .
\end{aligned}
$$

Here, the $G_{k \mu}$ connections are dynamical fields while $L_{0 \mu}$ and $R_{K+1 \mu}$ (appearing in the $\nabla_{\mu}$ operator acting on $\Sigma_{0,1}$ and $\left.\Sigma_{K, K+1}\right)$ are the sources enabling us to define the Noether currents of the chiral SU $(2)_{L_{0}} \times \mathrm{SU}(2)_{R_{K+1}}$ symmetry of this model. We thus get at this order exactly the same lagrangian as is assumed in the case of dimensional deconstruction, and the property of locality along the linear moose is also evident. The interest of this approach has to do with the additional terms at non-leading order in powers of $\epsilon$ : using the spurion expansion, we are in a position to determine which terms will appear next. Indeed the number of spurions required to turn the terms allowed by the $S_{\text {reduced, } K}$ symmetry into terms invariant under the full $S_{\text {natural }, K}$ symmetry gives us the order at which the corresponding term should appear. We now briefly describe these additional terms.

\subsubsection{Structure of the effective lagrangian}

Having described the spurion formalism to be used in the context of effective theories, we now go back to the problem of writing down the most general lagrangian satisfying our requirements of symmetry: invariance under $S_{\text {reduced, } K}$. If we now add the requirement of naturalness, our previous discussions show that this implies invariance under $S_{\text {natural }, K}$ and therefore involves spurions.

In order to write the most general effective lagrangian, we will use the fact that all terms which are invariant under the full $S_{\text {natural }, K}$ symmetry qualify as terms in our effective lagrangian. Such terms may be found by forming suitable combinations of building blocks which are covariant under the chiral transformations $\prod_{k=0}^{K} \mathrm{SU}(2)_{L_{k}} \times$ $\mathrm{SU}(2)_{R_{k+1}}$ acting on the Goldstone fields. One can then also apply covariant derivatives. Examples of such building blocks are

$$
\begin{aligned}
X_{k} Y_{k} & \longmapsto R_{k} X_{k} Y_{k} L_{k}^{\dagger}, \\
X_{k} G_{k \mu \nu} Y_{k} & \longmapsto R_{k} X_{k} G_{k \mu \nu} Y_{k} L_{k}^{\dagger} .
\end{aligned}
$$

We are also interested in classifying the terms in this lagrangian, following a double expansion: the usual momentum expansion and simultaneously an expansion in powers of spurions. After we have written down the full lagrangian, we may 'reduce' the terms by going to our standard gauge as described in section 2.2.1 and then inject the solution to the constraints to see what the dynamical content of the new terms is. One then obtains the constrained lagrangian, which does not involve the full spurions but rather the constants $\xi_{k}$ and $\eta_{k}$. The power counting is then provided, in addition to the expansion in momenta and gauge coupling-constants, by the powers of $\xi_{k}$ and $\eta_{k}$, that is, by the powers of $\epsilon$.

We have already written down in (2.41) the leadingorder terms in the lagrangian, which are the terms of or$\operatorname{der} \mathcal{O}\left(p^{2} \epsilon^{0}\right)$. Those terms do not involve spurions. We now discuss the next terms: once the constraints are applied, the terms that would appear at $\mathcal{O}\left(p^{2} \epsilon^{2}\right)$ are found to be constants or simple renormalizations of $\mathcal{O}\left(p^{2} \epsilon^{0}\right)$ terms since we have

$$
X_{k} X_{k}^{\dagger}=\xi_{k}^{2} \mathbb{1}_{2 \times 2}
$$




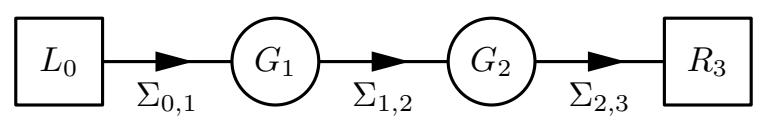

Fig. 2.3. The 'reduced' diagram for the model depicted in Fig. 2.2 after imposing the constraints.

and likewise for $Y_{k}$. On the other hand, there are new terms at $\mathcal{O}\left(p^{2} \epsilon^{4}\right)$. These yield the following terms in the standard gauge used in section 2.2.1

$$
\begin{aligned}
& \left\langle D_{\mu} \Sigma_{k, k+1} X_{k+1} Y_{k+1}\right. \\
\times & \left.D^{\mu} \Sigma_{k+1, k+2} \Sigma_{k+1, k+2}^{\dagger} Y_{k+1}^{\dagger} X_{k+1}^{\dagger} \Sigma_{k, k+1}^{\dagger}\right\rangle\left.\right|_{\text {const. }} \\
= & \xi_{k+1}^{2} \eta_{k+1}^{2}\left\langle\nabla_{\mu} \Sigma_{k, k+1}\right. \\
\times & \left.\nabla^{\mu} \Sigma_{k+1, k+2} \Sigma_{k+1, k+2}^{\dagger} \Sigma_{k, k+1}^{\dagger}\right\rangle
\end{aligned}
$$

and

$$
\begin{aligned}
& \left.\left\langle G_{k \mu \nu} Y_{k} \Sigma_{k, k+1} X_{k+1} G_{k+1}^{\mu \nu} X_{k+1}^{\dagger} \Sigma_{k, k+1}^{\dagger} Y_{k}^{\dagger}\right\rangle\right|_{\text {const. }} \\
= & \xi_{k+1}^{2} \eta_{k}^{2}\left\langle G_{k \mu \nu} \Sigma_{k, k+1} G_{k+1}^{\mu \nu} \Sigma_{k, k+1}^{\dagger}\right\rangle
\end{aligned}
$$

There are in fact other terms that would involve only products of spurions and Goldstone boson matrices without derivatives. Such terms would be non-leading in $\epsilon$, but of order $\mathcal{O}\left(p^{0}\right)$. However, upon going to the abovementioned gauge, such terms yield constant numbers as a consequence of the identity (2.44).

As we will see, the terms in (2.45) and (2.46) have important implications regarding the properties of the model: they yield corrections to the WSRs. Regarding symmetries and naturalness, we emphasize once again that the constrained lagrangian (2.41) is not invariant under the full original symmetry $S_{\text {natural, } K}$, but only under the $S_{\text {reduced, } K}$ subgroup, since the transformations $L_{k}$ and $R_{k}$ have been identified with $G_{k}$ for $k=1, \cdots, K$. Thus, there is no reason why the terms in the right-hand side of (2.45) and (2.46) should not be included. Since they are invariant under this reduced symmetry, they will in fact be required as counter-terms if we consider loops. Our formalism involving spurions shows how these terms may be consistently treated as being of higher order in an expansion around the lagrangian (2.41).

\subsection{Field redefinitions and unitary gauge}

The model is the stage of multiple Higgs mechanisms: $K$ gauge fields get masses and $K$ multiplets of Goldstone bosons disappear from the spectrum. The mass matrix for the gauge fields has a very specific structure, due to the nearest-neighbor interactions, and this will have important consequences later on. Counting the number of scalar fields, one notices that there remains a physical Goldstone multiplet in the spectrum. In order to describe the Higgs mechanism, we will perform a gauge-independent change of variables. We will refer to these field redefinitions by the phrase 'going to the unitary gauge', due to the similarity in the resulting lagrangian, but as a matter of fact, the procedure does not involve gauge-fixing [28,29]. We will give the appropriate field redefinitions to be performed in the full lagrangian but will then only write the resulting constrained lagrangian in order to avoid unnecessarily complicated equations.

We first define the unitary matrix $U$ describing the Goldstone bosons remaining in the spectrum, the normalization being fixed by the requirement that it be unitary. This implies that the multiplicative factors $\xi_{k}$ and $\eta_{k}{ }^{3}$ drop out from the definition

$$
\begin{aligned}
U & =\Sigma_{0,1} U_{1}\left(\prod_{j=1}^{K-1} V_{j} \Sigma_{j, j+1} U_{j+1}\right) \\
& \times V_{K} \Sigma_{K, K+1}
\end{aligned}
$$

which merely contains the angular parts $U_{k}$ and $V_{k}$ of the spurion fields as introduced in (2.28) and (2.29). We then perform the change of variable from $\Sigma_{0,1}$ to $U$. Next, we define the vector fields $W_{k}^{\mu}$ by the following relation, for $k=1, \cdots, K$

$$
\begin{aligned}
g_{k} W_{k}^{\mu} & =\mathrm{i}\left(\Sigma_{0,1} U_{1} \prod_{j=1}^{k-1} V_{j} \Sigma_{j, j+1} U_{j+1}\right) \\
& \times D^{\mu}\left(\left(\prod_{j=1}^{k-1} U_{j+1}^{\dagger} \Sigma_{j, j+1}^{\dagger} V_{j}^{\dagger}\right) U_{1}^{\dagger} \Sigma_{0,1}^{\dagger}\right) \\
& -\mathrm{i} \alpha_{k} U D^{\mu} U^{\dagger}
\end{aligned}
$$

and perform the change of variables from the $G_{k}^{\mu}$ fields to the $W_{k}^{\mu}$ fields. From the transformation properties of $U_{k}$ and $V_{k}$, we know that the covariant derivatives appearing in this equation are given by

$$
\begin{aligned}
D_{\mu} U_{k} & =\partial_{\mu} U_{k}-\mathrm{i} R_{k \mu} U_{k}+\mathrm{i} g_{k} U_{k} G_{k \mu}, \\
D_{\mu} V_{k} & =\partial_{\mu} V_{k}-\mathrm{i} g_{k} G_{k \mu} V_{k}+\mathrm{i} V_{k} L_{k \mu},
\end{aligned}
$$

which identically vanish when the constraints are imposed on the spurions. The coefficients $\alpha_{k}$ in (2.48) are defined by

$$
\alpha_{k}=f_{\pi}^{2} \sum_{j=0}^{k-1} \frac{1}{f_{j}^{2}}, \text { for } k=1, \cdots, K
$$

where we have introduced

$$
\frac{1}{f_{\pi}^{2}}=\sum_{j=0}^{K} \frac{1}{f_{j}^{2}} .
$$

3 We remind the reader that $\xi_{k}$ and $\eta_{k}$ are at this stage functions: we are writing down the fields redefinitions in a general gauge and independently of the constraints. 
The transformation properties of the newly defined fields are as follows

$$
\begin{aligned}
U & \longmapsto L_{0} U R_{K+1}^{\dagger}, \\
W_{k \mu} & \longmapsto L_{0} W_{k \mu} L_{0}^{\dagger}, \text { for } k=1, \cdots, K,
\end{aligned}
$$

and when we then perform the change of variables according to

$$
\begin{aligned}
& \left\{G_{1 \mu}, \cdots, G_{K \mu}, \Sigma_{0,1}, \cdots, \Sigma_{K, K+1}\right\} \\
\longrightarrow & \left\{W_{1 \mu}, \cdots, W_{K \mu}, U, \Sigma_{1,2}, \cdots, \Sigma_{K, K+1}\right\} .
\end{aligned}
$$

We find that, as a consequence of the symmetries, the lagrangian written in terms of these variables does not depend on $\Sigma_{1,2}, \ldots, \Sigma_{K, K+1}$ : this is the Higgs mechanism. In addition, the choice (2.51) for the coefficients $\alpha_{k}$ eliminates all mixing between the Goldstone bosons $U$ and the vector fields $W_{k \mu}$.

It is in fact sufficient at this stage to work directly with the constrained form of the lagrangian. With this in mind, we give the constrained form of the field redefinitions in order to display the field content in a more intuitive way

$$
\begin{aligned}
&\left.U\right|_{\text {const. }}=\prod_{j=0}^{K} \Sigma_{j, j+1} \\
&\left.g_{k} W_{k}^{\mu}\right|_{\text {const. }}=\mathrm{i}\left(\prod_{j=0}^{k-1} \Sigma_{j, j+1}\right) D^{\mu}\left(\prod_{j=0}^{k-1} \Sigma_{j, j+1}\right)^{\dagger} \\
&-\left.\mathrm{i} \alpha_{k} U D^{\mu} U^{\dagger}\right|_{\text {const. }} \\
& \text { for } k=1, \cdots, K .
\end{aligned}
$$

Upon rewriting the lagrangian in terms of the new variables, one then recognizes $f_{\pi}$, defined in (2.52), as the Goldstone boson decay constant.

In addition, the entry in the $k$-th row and $k^{\prime}$-th column of the mass-squared matrix $\mathcal{M}^{2}$ for the $W_{k}^{\mu}$ fields are given by

$$
\begin{aligned}
{\left[\mathcal{M}^{2}\right]_{k, k^{\prime}} } & =\frac{1}{4} \delta_{k, k^{\prime}} g_{k}^{2}\left(f_{k-1}^{2}+f_{k}^{2}\right) \\
& -\frac{1}{4}\left(\delta_{k, k^{\prime}+1}+\delta_{k+1, k^{\prime}}\right) g_{k} g_{k+1} f_{k}^{2}
\end{aligned}
$$

for $k, k^{\prime}=1, \cdots, K$. The elements of this mass matrix are of order $\mathcal{O}\left(p^{2} \epsilon^{0}\right)$ since they contain two powers of gauge couplings. This entails that the masses of the vector bosons have to be considered small in some sense in our expansion. In practice, this has to be understood as follows: the corresponding fields really belong to the LEET only if their masses are smaller than the scale at which the low-energy perturbation expansion breaks down and at which other resonances have to be taken into account. We will not discuss the issue of estimating the value of this scale. Suffices to say here that the information that can be gleaned from the LEET containing the Goldstone modes only $30,31,32$, points to a value in the vicinity of $4 \pi f_{\pi}$, but that there is a debate over these matters [33], due to the restoration of tree unitarity from Kaluza-Klein excitations of gauge fields in the extra-dimensional case [34 35], or from the very resonances we are considering here or in the dimensional deconstruction view. Coming back to our main line of discussion, we notice that the only non-zero entries in the mass-squared matrix (2.58) are on the diagonal and just above or just below it. This structure entails that the first powers of this matrix have zero coefficient in the off-diagonal corners, that is

$$
\left[\left(\mathcal{M}^{2}\right)^{l-1}\right]_{1, K}=0, \text { for } l=1, \cdots, K-1 .
$$

On the other hand, the first inverse power of this matrix is such that it satisfies the relation

$$
\frac{g_{1} g_{K} f_{0}^{2} f_{K}^{2}}{4}\left[\left(\mathcal{M}^{2}\right)^{-1}\right]_{1, K}-f_{\pi}^{2}=0
$$

as can be calculated explicitly by inference on $K$, for $K \geqslant$ 1. We will make use of relations (2.59) and (2.60) later on in section 3.1 Note that in sections 4 and 5 we will be coupling gauge fields at the ends of the chain, and also connecting the two ends. This has no influence on the mass matrix for the $W_{k \mu}$ fields, but only on the kinetic terms for these fields. In order to bring the lagrangian to the canonical form, further field redefinitions involving rescaling of fields will then be required.

\section{The left-right two-point correlator}

The constrained moose shown in Fig. 2.3 represents a chain of $K$ gauge fields $G_{k \mu}$ interacting with $K+1$ Goldstone boson multiplets $\Sigma_{k, k+1}$, where $K=2$ in the figure. As a consequence of the dynamical Higgs mechanism, the latter disappear from the spectrum but for one combination parametrized by $U$. This gives the mass matrix (2.58) to the vectors.

The characteristic long-distance feature of the model is its global chiral symmetry $\mathrm{SU}(2)_{L_{0}} \times \mathrm{SU}(2)_{R_{K+1}}$, which is spontaneously broken, generalizing the usual case of the non-linear sigma model $(K=0)$. The Noether currents generating this symmetry may be obtained by taking the functional derivative with respect to the sources $L_{0 \mu}$ and $R_{K+1 \mu}$ in (2.41). However, the lagrangian (2.41) is endowed with additional interesting short-distance properties which are reminiscent of QCD, despite the model bearing no obvious resemblance to any QCD-like theory.

Indeed, in QCD, the spontaneously broken chiral symmetry combines with the operator product expansion (OPE) and the operator content of the theory to yield the two Weinberg sum rules [16]: the two-point correlator of lefthanded and right-handed Noether currents transforms according to the $(3,3)$ representation of the chiral $\mathrm{SU}(2) \times$ $\mathrm{SU}$ (2) group and there are no corresponding local operators in the theory with mass-dimension strictly lower than six 36. Consequently, the left-right correlator behaves smoothly at short distances, leading among other things to the finite electroweak mass of the pseudo-Goldstone bosons (pions) 37. 
It has already been pointed out [17 that the lagrangian (2.41) also leads to the two Weinberg sum rules, rendering the theory smoother at high energies than naively expected: we stress that this is in fact at the origin of the little Higgs phenomenon. However, as is the case in QCD, this feature is not a consequence of low-energy symmetries characteristic of the lagrangian (2.41). What is crucial in addition is the property of locality along the chain: the fact that only nearest-neighbors interact. This property is emphasized when the lagrangian (2.41) is rewritten in the form

$$
\begin{aligned}
\left.\mathcal{L}^{(2,0)}\right|_{\text {const. }} & =\mathcal{L}_{0}\left(L_{0 \mu}, \Sigma_{1,0}, G_{1 \mu}\right) \\
& +\mathcal{L}_{K}\left(G_{K \mu}, \Sigma_{K, K+1}, R_{K+1 \mu}\right) \\
& +\sum_{k=1}^{K-1} \mathcal{L}_{k}\left(G_{k \mu}, \Sigma_{k, k+1}, G_{k+1 \mu}\right)
\end{aligned}
$$

We then see that, in order to connect the left and right sources $L_{0 \mu}$ and $R_{K+1 \mu}$, one has to perform at least $K$ contractions, pushing the left-right correlator to higher orders. This property of the theory indeed does not follow from the symmetry $S_{\text {reduced,K }}$ of (2.41) alone: one needs either to invoke dimensional deconstruction and locality in the fifth dimension, or to use the higher symmetry $S_{\text {natural, } K}$ and the corresponding spurion formalism. Terms which are not of the form (2.41) and which break the Weinberg sum rules are indeed part of the theory, but are of higher order in $\epsilon$.

In this section, these statements are further clarified and extended: we are going to show that, at $\operatorname{order} \mathcal{O}\left(p^{2} \epsilon^{0}\right)$, the theory actually contains $K$ Weinberg sum rules generalizing those known from QCD.

\subsection{Generalized Weinberg sum rules}

The claims made above concerning the left-right correlator pertain to the two-point function of the Noether currents corresponding to the symmetry under which the Goldstone multiplet remaining in the spectrum transforms. Recalling the transformation properties of $U$ (2.53), we now focus on the second derivative of the generating functional with respect to the sources $L_{0 \mu}$ and $R_{K+1 \mu}$ : this study is performed at lowest-order and consequently at tree-level. We define the function $\Pi_{L R}$ through the relation

$$
\begin{aligned}
& 4 \mathrm{i} \int \mathrm{d} x \mathrm{e}^{\mathrm{i} q \cdot x}\left\langle 0\left|T J_{L_{0}}^{a \mu}(x) J_{R_{K+1}}^{b \nu}(0)\right| 0\right\rangle \\
= & -\delta^{a b}\left(\eta^{\mu \nu} q^{2}-q^{\mu} q^{\nu}\right) \Pi_{L R}\left(-q^{2}\right) .
\end{aligned}
$$

One may evaluate this by taking the second derivative of the path integral with respect to the sources and then evaluating the tree-level diagrams in the basis of vector fields which diagonalizes the mass matrix (2.58). We will however follow another route, which displays in a more transparent fashion the origin of the relations we are interested in, using the $W_{k}^{\mu}$ fields.

We first extract the expressions for the currents in terms of the $W_{k \mu}$ fields: this is easily done by first taking the functional derivatives of the original expression for the lagrangian (2.41), and then performing the change of variables implied by the definitions (2.57) and (2.56). Again, we work with the constrained lagrangian in order to keep the equations as simple as possible. We find the expected field-current identities 38 39,14, with an additional term involving the remaining Goldstone bosons

$$
\begin{gathered}
J_{L_{0}}^{a \mu}=\left.\frac{\delta \mathcal{L}_{2}}{\delta L_{0 \mu}^{a}}\right|_{L_{0 \rho}=0, R_{K+1 \sigma}=0} \\
=-\frac{g_{1} f_{0}^{2}}{4} W_{1}^{a \mu}-\mathrm{i} \frac{f_{\pi}^{2}}{4}\left\{U \partial^{\mu} U^{\dagger}\right\}^{a}, \\
J_{R_{K+1}}^{a \mu}=\left.\frac{\delta \mathcal{L}_{2}}{\delta R_{K+1 \mu}^{a}}\right|_{L_{0 \rho}=0, R_{K+1 \sigma}=0} \\
=-\frac{g_{K} f_{K}^{2}}{4}\left\{U^{\dagger} W_{K}^{\mu} U\right\}^{a} \\
-\mathrm{i} \frac{f_{\pi}^{2}}{4}\left\{U^{\dagger} \partial^{\mu} U\right\}^{a} .
\end{gathered}
$$

The presence of the matrix $U$ in the second line of 3.4 follows from the lack of symmetry of our definitions (2.57) relative to the center of the chain. This is a minor flaw, and we stick to these definitions based on their simplicity.

Only the terms linear in fields in the above expressions for the currents are relevant for the evaluation of the twopoint function at tree level. The only contributions in this model come from Goldstone boson and massive resonance exchange, and we find at this order

$$
\begin{aligned}
& 4 \mathrm{i} \int \mathrm{d} x \mathrm{e}^{\mathrm{i} q \cdot x}\left\langle 0\left|T J_{L_{0}}^{a \mu}(x) J_{R_{K+1}}^{b \nu}(0)\right| 0\right\rangle \\
= & \delta^{a b} \frac{g_{1} g_{K} f_{0}^{2} f_{K}^{2}}{4} \\
\times & {\left[\left(\eta^{\mu \nu} \mathbb{1}-q^{\mu} q^{\nu}\left(\mathcal{M}^{2}\right)^{-1}\right)\left(q^{2} \mathbb{1}-\mathcal{M}^{2}\right)^{-1}\right]_{1, K} } \\
+ & \delta^{a b} f_{\pi}^{2} \frac{q^{\mu} q^{\nu}}{q^{2}} .
\end{aligned}
$$

Expanding this for large euclidean momenta $Q^{2}=-q^{2} \rightarrow$ $+\infty$ yields

$$
\begin{aligned}
\Pi_{L R}\left(Q^{2}\right) & =-\frac{g_{1} g_{K} f_{0}^{2} f_{K}^{2}}{4} \\
& \times \sum_{l=1}^{\infty} \frac{\left[\left(-\mathcal{M}^{2}\right)^{l-1}\right]_{1, K}}{Q^{2(l+1)}} .
\end{aligned}
$$

Note that the $l=0$ term in this equation is absent for $K \geqslant$ 1 , due to the relation (2.60) (compare expressions (3.5) and (3.6)). This is the first Weinberg sum rule, which is valid here at tree-level: the first moment in the expansion of $\Pi_{L R}$ at large $Q^{2}$ is zero. In this model where the contributions to the correlator come from infinitely narrow resonances, the Weinberg sum rules are simply relations between the masses of the resonances and the decay constants of the Goldstone bosons and resonances as 
we shall see in section 3.2 Note that relation (2.60) can also be deduced from expression (3.5) and the transversity of the two-point function, which is itself a consequence of current-conservation. From the relations (2.59), we see that the terms with $l=1, \cdots, K-1$ in the infinite series in the right-hand-side of (3.6) are also zero. This gives us additional Weinberg sum rules. The case $l=1$ is recognized as the second Weinberg sum rule. Thus, the case of a chain with two intermediate sites $(K=2)$ reproduces exactly two Weinberg sum rules.

In the general case, equation (3.6) shows that the twopoint function $\Pi_{L R}$ decreases for large euclidean momenta as $1 / Q^{2(K+1)}$ and not as $1 / Q^{2}$, where $K$ is the number of internal sites in the chain. This in turn implies that the $K$ first operators appearing as coefficients in the expansion (3.6), which are order parameters of the chiral symmetry, are found to have a vanishing vacuum expectation value in such models at this order. We have thus related the smooth high-energy behavior of moose models to an extension of a well-known property of QCD.

\subsection{Other aspects}

In this section, we point out other relevant aspects of the left-right correlator which require a closer inspection of the algebra involved with the diagonalization of the mass matrix. In fact, it turns out that we can learn more about the left-right correlator, even without explicitly diagonalizing the mass matrix, but just using the orthogonality properties of the transformation matrix to the diagonal basis.

As already mentioned, an alternative writing for $\Pi_{L R}$ uses the basis in which the mass matrix is diagonal: this proceeds through the definition of the $A_{n}^{\mu}$ fields [17.

$$
A_{n}^{\mu}=\sum_{k=1}^{K} b_{n}^{k} W_{k}^{\mu}, \text { for } n=1, \cdots, K,
$$

where the orthogonal matrix $b$ is as yet unknown. We denote by $M_{n}^{2}$ the mass squared of the field $A_{n}^{\mu}$. These masses as well as the $b_{n}^{k}$ coefficients may be calculated explicitly only in particular cases ${ }^{4}$. Taking the second functional derivative with respect to the sources $L_{0 \mu}, R_{K+1 \nu}$ of the generating functional defined by the path-integral over the lagrangian (2.41), we get at tree-level

$$
\Pi_{L R}\left(Q^{2}\right)=-\frac{f_{\pi}^{2}}{Q^{2}}+\sum_{n=1}^{K} \frac{F_{n}^{2}}{Q^{2}+M_{n}^{2}},
$$

where the coupling constant $F_{n}^{2}$ of the massive vector field $A_{n}^{\mu}$ is given by

$$
F_{n}^{2}=\frac{1}{4} g_{1} g_{K} f_{0}^{2} f_{K}^{2} \frac{b_{n}^{1} b_{n}^{K}}{M_{n}^{2}} .
$$

\footnotetext{
${ }^{4}$ We will work out an example explicitly in section 3.3
}

Explicit calculation of the quantities

$$
\begin{aligned}
\sum_{n=1}^{K} F_{n}^{2}-f_{\pi}^{2} & =0, \\
\sum_{n=1}^{K} F_{n}^{2} M_{n}^{2(l-1)} & =0, \text { for } l=2, \cdots, K,
\end{aligned}
$$

using (3.9) then gives an alternative derivation of the $K$ Weinberg sum rules of section 3.1

Depending on the sign of the constant $F_{n}^{2}$, one may call the corresponding $n$-th vector field a vector or an axial resonance even though the names do not apply in the strict sense since the chain is not necessarily symmetric under reflection with respect to its center - 'parity' ${ }^{5}$. In this manner, one may determine the first non-zero moment in the expansion (3.6)

$$
\begin{aligned}
\Pi_{L R}\left(Q^{2}\right) & =-\left(\prod_{i=1}^{K} g_{i}^{2}\right)\left(\prod_{j=0}^{K} \frac{f_{j}^{2}}{4}\right) \frac{1}{Q^{2(K+1)}} \\
& +\mathcal{O}\left(\frac{1}{Q^{2(K+2)}}\right) .
\end{aligned}
$$

Thus, in the limit of large $Q^{2}$ we have $Q^{2} \Pi_{L R}\left(Q^{2}\right)<0$, as implied in the case of QCD by the inequality derived by Witten 41. This result once again confirms that of [40] which considered resonances independently of a lagrangian model, but assuming invariance under parity.

We briefly turn to the low-energy consequences of our model: here again, we do not need to know the diagonalization for the mass matrix explicitly, just its properties.

By integration of the massive resonances, one may in principle derive the values of the LECs for the chiral lagrangian corresponding to this model. Integration of the resonances require that they be parametrically heavier than the remaining particles. In our case, even though the masses of the resonances are small parameters in the sense of our expansion (they are counted as $\mathcal{O}\left(p^{2} \epsilon^{0}\right)$ ), the remaining particles are the massless Goldstone bosons, and the procedure is thus meaningful. As a simple application, one may determine the constant $L_{10}$ defined by Gasser and Leutwyler [4, by making use of the equations of motion for the resonances [21], or directly through the following relation [3]

$$
\begin{aligned}
L_{10} & =-\frac{1}{4} \int_{0}^{+\infty} \mathrm{d} s\left(\frac{1}{\pi} \operatorname{Im}\left(\Pi_{L R}(s)\right)+f_{\pi}^{2} \delta(s)\right) \\
& =-\frac{1}{4} \sum_{n=1}^{K} \frac{F_{n}^{2}}{M_{n}^{2}} .
\end{aligned}
$$

\footnotetext{
${ }^{5}$ It is possible to show quite generally that the signs for the $F_{n}^{2}$ alternate, starting with a vector resonance $\left(F_{1}^{2}>0\right)$. This was already noticed in [17] for the parity-invariant or symmetric moose. The authors of [40] also point out that this is the case in general whatever the model for resonances when one has exactly as many resonances as Weinberg sum rules.
} 
We find

$$
L_{10}=-\sum_{k=1}^{K} \frac{\alpha_{k}\left(1-\alpha_{k}\right)}{g_{k}^{2}}
$$

Note that the constants $\alpha_{k}$ appearing in this equation are known explicitly from (2.51) in the generic case. If we take the view that our moose model is to be used as a model for the low-energy sector of a QCD-type theory, then this constant $L_{10}$ is a non-local order parameter of chiral symmetry breaking: we observe that $L_{10}<0$ in this model. This fact will have repercussions later on in section 5 when we use these moose models as a basis for electroweak symmetry breaking: the constant $L_{10}$ then gives us the value of the $S$ parameter [6] since we are restricting ourselves to tree-level. We have seen that the sign of the parameter is fixed ${ }^{6}$, however, its magnitude can be made arbitrarily small if we set one of the decay constants at the end of the chain to be much smaller than the others. In practice, we would choose $f_{K} \ll f_{k}$ for $k=0, \cdots, K-1$ with the conventions to be adopted in section 4 and 5 One may also choose the gauge coupling constants to be large enough in order to avoid a large value of $L_{10}$ at tree-level but the freedom in this respect is limited given the power counting (2.9) for the gauge coupling constants $g_{k}$.

\subsection{Particular cases}

\subsubsection{Case with all decay constants equal and all gauge couplings equal}

Up to this point the derivations have been general and the results apply in all cases. Still, we are limited for practical applications as soon as $K$ is larger than a few units, since we are then unable to diagonalize the mass matrix and find the explicit values of the $b_{n}^{k}$ coefficients. One may want to derive further explicit results to get a feeling of what happens. We will do this in the simplest possible case while keeping $K$ undetermined: we pick the particular case where all decay constants are equal, and all gauge coupling constants are equal

$$
\begin{aligned}
& f_{k}=f, \text { for } k=0, \cdots, K, \\
& g_{k}=g, \text { for } k=1, \cdots, K
\end{aligned}
$$

We then derive the following masses for the $K$ resonances

$$
\begin{aligned}
& M_{n}^{2}=g^{2} f^{2} \sin ^{2}\left(\frac{\pi n}{2(K+1)}\right) \\
& \quad \text { for } n=1, \cdots, K
\end{aligned}
$$

and the following explicit expressions for the $F_{n}^{2}$

$$
\begin{aligned}
& F_{n}^{2}=\frac{2}{K+1}(-1)^{n+1} f^{2} \cos ^{2}\left(\frac{\pi n}{2(K+1)}\right), \\
& \quad \text { for } n=1, \cdots, K .
\end{aligned}
$$

\footnotetext{
${ }^{6}$ Changing the sign would require a model where there are fewer Weinberg sum rules than resonances, as can be seen from [40].
}

With this formula, we can explicitly verify that we have a tower of alternating vector and axial resonances ${ }^{7}$. The expression for $L_{10}$ in this particular case is found to be

$$
L_{10}=-\frac{1}{6 g^{2}} \frac{K(K+2)}{K+1} \text {. }
$$

\subsubsection{Case with $K=1$}

We give a short summary of relevant results for this simple case as a preparation for section 5.2.3 The study of the $K=1$ linear moose model is performed as in the general case presented above: there is only one triplet of massive vector fields $W_{1 \mu}^{a}, a=1,2,3$, with masses

$$
M_{W_{1}}^{2}=g_{1}^{2} \frac{f_{0}^{2}+f_{1}^{2}}{4} .
$$

The decay constant $f_{\pi}$ of the remaining Goldstone bosons is related to the original parameters in the lagrangian through relation (2.52), which becomes

$$
f_{\pi}^{2}=\frac{f_{0}^{2} f_{1}^{2}}{f_{0}^{2}+f_{1}^{2}}
$$

For the $K=1$ linear moose model, we have exactly one Weinberg sum rule, and (3.8) is rewritten

$$
\Pi_{L R}\left(Q^{2}\right)=-f_{\pi}^{2} M_{W_{1}}^{2} \frac{1}{Q^{2}\left(Q^{2}+M_{W_{1}}^{2}\right)}
$$

\subsection{Non-leading interactions and corrections to the WSRs}

We now briefly describe the effect of the non-leading terms in $\epsilon$ mentioned in (2.45) and (2.46). These additional interactions are of order $\mathcal{O}\left(p^{2} \epsilon^{4}\right)$, and thus only a limited number of them may occur at a given order in the expansion. For most of this paper we will not fix the relation between the counting in powers of $p$ and that in powers of $\epsilon$, since we want to be as general as possible.

In the dimensional deconstruction approach the terms we are considering here are omitted on the basis that they correspond to non-local interactions with respect to the fifth (deconstructed) dimension. However, we want to consider their effect since they are not forbidden by the symmetries of the problem once we have introduced the gauge interactions, but only suppressed in our power counting scheme. In any case, they will be produced by radiative corrections.

\footnotetext{
7 This time, the names apply in the strict sense since we are considering a symmetric moose.
} 
We first consider the following lagrangian, where $\mathcal{O}\left(p^{2} \epsilon^{4}\right)$ terms of the type (2.45) have been included

$$
\begin{aligned}
\left.\mathcal{L}^{\prime}\right|_{\text {const. }} & =\frac{1}{4} \sum_{k=0}^{K} f_{k}^{2}\left\langle\nabla_{\mu} \Sigma_{k, k+1} \nabla^{\mu} \Sigma_{k, k+1}^{\dagger}\right\rangle \\
& -\frac{1}{2} \sum_{k=1}^{K}\left\langle G_{k \mu \nu} G_{k}^{\mu \nu}\right\rangle \\
& +\frac{1}{2} \sum_{k=0}^{K-1} \xi_{k+1}^{2} \eta_{k+1}^{2} f_{k, k+1}\left\langle\nabla_{\mu} \Sigma_{k, k+1} \nabla^{\mu} \Sigma_{k+1, k+2}\right. \\
& \left.\times \Sigma_{k+1, k+2}^{\dagger} \Sigma_{k, k+1}^{\dagger}\right\rangle .
\end{aligned}
$$

If we derive the expression for the two-point left-right function implied by this lagrangian at tree-level and up to order $\mathcal{O}\left(p^{2} \epsilon^{4}\right)$, we find that the last Weinberg sum rule obtains a correction, (the $l=K$ equation in (3.11) then includes an $\mathcal{O}\left(p^{2(K-1)} \epsilon^{4}\right)$ term in the right-hand side whereas individual terms in left-hand side are of or$\left.\operatorname{der} \mathcal{O}\left(p^{2(K-1)} \epsilon^{0}\right)\right)$. This is expected from the naive observation that the effective length of the chain is reduced: we indeed have interactions involving two neighboring links. This may be rephrased along the same lines as the reasoning following (3.1): the number of gauge interactions required to have a non-zero correlation between left and right currents is reduced. Indeed, linear combinations of terms of the type

$$
\left\langle\nabla_{\mu} \Sigma_{k, k+1} \nabla^{\mu} \Sigma_{k, k+1}^{\dagger}\right\rangle
$$

and

$$
\left\langle\nabla_{\mu} \Sigma_{k, k+1} \nabla^{\mu} \Sigma_{k+1, k+2} \Sigma_{k+1, k+2}^{\dagger} \Sigma_{k, k+1}^{\dagger}\right\rangle,
$$

can be recast as combinations of

$$
\left\langle\nabla_{\mu} \Sigma_{k, k+1} \nabla^{\mu} \Sigma_{k, k+1}^{\dagger}\right\rangle,
$$

and

$$
\begin{gathered}
\left\langle\nabla_{\mu}\left(\Sigma_{k, k+1} \Sigma_{k+1, k+2}\right)\right. \\
\left.\times \nabla^{\mu}\left(\Sigma_{k+1, k+2}^{\dagger} \Sigma_{k, k+1}^{\dagger}\right)\right\rangle .
\end{gathered}
$$

The point is that the right transformation of $\Sigma_{k, k+1}$ is identified with the left transformation of $\Sigma_{k+1, k+2}$, and the corresponding interactions come in without an additional power of gauge coupling constant. This description is reminiscent of the standard motivation for little Higgs models 24 .

Coming back to our discussion of the low-energy constant $L_{10}$ 3.14), we note that, provided the spurion term in 3.23 represents a small correction, one expects

$$
\xi_{k+1}^{2} \eta_{k+1}^{2}\left|f_{k, k+1}\right| \ll f_{j}^{2}
$$

which in turn implies that $L_{10}$ remains negative.
If one now considers the additional terms involving gauge field curvatures as shown in (2.46), then all Weinberg sum rules but the first one are affected by the presence of $\mathcal{O}\left(p^{2} \epsilon^{4}\right)$ terms in the lagrangian: the particular sums of $\mathcal{O}\left(p^{2(l-1)} \epsilon^{0}\right)$ constants on the left-hand side of (3.11) are evaluated to be of order $\mathcal{O}\left(p^{2(l-1)} \epsilon^{4}\right)$. This comes about because the diagonalization of the kinetic terms for the vector fields is modified. The first Weinberg sum rule on the other hand, being related to the transversity of the two-point left-right function remains valid. In fact, it is possible to destroy this sum rule as well, provided we introduce terms similar to (2.45) involving the sources $^{8}$

$$
\begin{gathered}
\left\langle L_{0 \mu \nu} \Sigma_{0,1} X_{1} G_{1}^{\mu \nu} X_{1}^{\dagger} \Sigma_{0,1}^{\dagger}\right\rangle \\
\left\langle G_{K \mu \nu} Y_{K} \Sigma_{K, K+1} R_{K+1}^{\mu \nu} \Sigma_{K, K+1}^{\dagger} Y_{K}^{\dagger}\right\rangle .
\end{gathered}
$$

However, such terms only appear at order $\mathcal{O}\left(p^{3} \epsilon^{2}\right)$ : for them to appear at a lower order, they would need to be divided by a gauge coupling constant, making them illdefined in the limit $g_{k} \longrightarrow 0$. The same would then be true of the Noether currents of the theory, even though the contribution to the charge would be zero, being a surface term. Therefore, the requirement that the off-shell Noether currents of the theory be well-defined in this limit forbids these terms at order $\mathcal{O}\left(p^{2} \epsilon^{2}\right)$ and implies that the first Weinberg sum rule could at most be violated starting at order $\mathcal{O}\left(p^{3} \epsilon^{2}\right)$.

In this section, we have seen that the WSRs get modified by terms of higher powers in the expansion. To the extent that the $\xi_{k}$ and $\eta_{k}$ constants are small parameters, these modifications represent small corrections: the righthand side of (3.11 no more vanishes, but is suppressed by a factor of order $\mathcal{O}\left(p^{0} \epsilon^{4}\right)$ relative to individual terms in the left-hand side. This is similar to the case of QCD, where the second WSR is corrected due to non-zero quark masses, which in $\chi \mathrm{PT}$ are also viewed as spurions.

\subsection{Discussion}

We have seen in section 3.1 that imposing naturalness in the sense of the spurion formalism of section 2 2 on an open linear moose model with $K$ internal sites implies $K$ Weinberg sum rules at lowest order. At next order, these sum rules receive corrections as discussed in section 3.4 This means that in the underlying model whose LEET we have been constructing, the order parameters which give the coefficients in the expansion (3.6) are naturally small. Now the particular case where a given number of sum rules are valid has been studied in [40, where the coupling constants and masses of the vector and axial resonances are studied assuming parity. What we have displayed here is

8 This does not conflict with the transversity argument just alluded to because in this case, there are additional local contributions to the two-point left-right function which destroy the link between the first Weinberg sum rule and the transversity of this two-point function. 
not the full theory either, but a possible lagrangian for the low-energy sector, which means that we are in a position to discuss other Green's functions, not only two-point ones. Our model is only one particular instance realizing a given number of Weinberg sum rules within some approximation, but it might nonetheless be useful in conjunction with the large- $N_{c}$ expansion of QCD [42,43,44. In view of such applications, one would only be interested in the case $K=1$ or $K=2$.

Other cases with a longer moose would give us lowenergy descriptions for theories where the chiral symmetry breaking pattern is different from that of QCD, suppressing the order parameters appearing in the expansion (3.6). Even though such models do not seem to be relevant to $\mathrm{QCD}$, they might be helpful in understanding the various patterns of chiral symmetry breaking. In particular, one would be interested in knowing in which respects the underlying theory would differ from QCD if such a comparison is possible. However, such an endeavor is to be carried out outside the domain of applicability of the effective theory we have built.

\section{Minimal model of EWSB}

As already mentioned in section 3 the case of the linear moose with $K=0$ corresponds to the non-linear sigma model and can be viewed as the minimal symmetrybreaking sector for the electroweak gauge theory: it only contains the three Goldstone modes required to give masses to the $W^{ \pm}$and $Z^{0}$ vector bosons and no additional particles, in particular no physical Higgs boson. Even though the corresponding lowest-order lagrangian has been used to tackle the heavy-Higgs limit of the SM in 9, 10 11 and more systematically in [12], we wish to emphasize that in general there need not be an underlying scalar resonance to replace the Higgs and that the approach is indeed much more general. In this section, we focus on the simplest LEET for the electroweak sector where the Higgs mechanism occurs dynamically, that is to say, where there is no physical Higgs boson in the spectrum as a remnant of a complex doublet ${ }^{9}$.

The point of view of the LEET framework is to construct the effective lagrangian based on the assumed symmetries of the underlying theory - which we will call 'technitheory' - with as little reference as possible to its details. The denomination techni-theory stems from the known fact that the effective lagrangian [6] for technicolor [45] and other alternative symmetry-breaking sectors is built around the non-linear sigma model whereas the generalizations we will be dealing with are based on extensions of the moose. In fact, the authors of [46] already noticed that moose models encompass many cases of dynamical electroweak symmetry breaking.

A typical difficulty of technicolor theories (requiring the introduction of extended technicolor) is the problem

\footnotetext{
${ }^{9}$ Or equivalently, as the radial component of a two-by-two matrix $\Sigma$ satisfying a reality condition $\Sigma^{c}=\Sigma$, see section 2.2 .1
}

of fermion mass generation [47] and of the presence of anomalous couplings for these fermions [48,49]. This last point is also true of BESS models [50 51]. We will see that, although the LEET does not deal with the origin of the constraints applied on the space of gauge configurations, it provides a rationale for the classification of their effects through the use of spurions, enabling us to discuss whether or not a given term may appear in the lagrangian at leading order. It also shows that any theory of dynamical EWSB a priori involves enough weak isospin breaking to provide mass-splittings within fermion doublets. In this section we discuss how the expansion in powers of spurions does address some of the long-standing questions we have mentioned.

We now describe the leading-order properties of the simplest model one can imagine for EWSB based on mooses: the low-energy description then only includes the electroweak gauge fields and the three Goldstone modes that are required to give masses to the $W^{ \pm}$and $Z^{0}$ bosons. In other words, the spectrum of the LEET does not include any scalars. This is a well-known case, but the interest of our formalism here mostly has to do with fermions: possible anomalous couplings are suppressed by powers of $\epsilon$. This is a situation where those terms allowed by the reduced symmetry of the theory are not allowed by the larger natural symmetry, and can therefore be consistently treated as being of higher order, the order being given by the number of spurions involved. Another prediction from our spurion formalism is that mass-splittings within the doublets automatically appear at the same order as the mean masses in the doublets, whereas these splittings have always been difficult to account for in traditional approaches to dynamical symmetry breaking. In addition, we note that the operators giving a tree-level $S$ parameter do not appear at leading order. All these results are accounted for by the same formalism, embodied by the spurion expansion, which was originally introduced with a different aim: to restore naturalness in a given limit.

This first model is based on the $K=0$ linear moose - which has an SU (2) $\times \mathrm{SU}(2)$ symmetry - but adds two dynamical SU(2) groups. Furthermore, we will introduce a U (1) $B-L$ group when we consider fermions. This means that the natural symmetry for this model, before it is reduced by the constraints imposed on the spurions, is

$$
S_{\text {natural }}=\mathrm{SU}(2)^{4} \times \mathrm{U}(1)_{B-L}
$$

Applying constraints on the spurions will enable us to reduce this symmetry to

$$
S_{\text {reduced }}=\mathrm{SU}(2) \times \mathrm{U}(1)_{Y} .
$$

The details of this reduction, as well as the physical consequences are the subject of this section. As we shall see later on in sections 5.1 and 5.2 many of the results still hold when we consider extensions of this minimal case. For this reason, the current section, being central to the paper, is fairly detailed. 


\subsection{Complex spurion and $U(1)_{\tau^{3}}$}

The model is obtained from the $K=0$ linear moose by coupling gauge fields to the only Goldstone multiplet as shown in Fig. 4.1 ${ }^{10}$. The two $\mathrm{SU}(2)$ gauge fields transform as

$$
\begin{gathered}
G_{0 \mu} \longmapsto G_{0} G_{0 \mu} G_{0}^{\dagger}+\frac{\mathrm{i}}{g_{0}} G_{0} \partial_{\mu} G_{0}^{\dagger}, \\
G_{1 \mu} \longmapsto G_{1} G_{1 \mu} G_{1}^{\dagger}+\frac{\mathrm{i}}{g_{1}} G_{1} \partial_{\mu} G_{1}^{\dagger},
\end{gathered}
$$

and the corresponding transformations commute with the chiral symmetry generated by $L_{0}$ and $R_{1}$.

The final selection of the $\mathrm{SU}(2) \times \mathrm{U}(1)$ gauge group that becomes dynamical follows from the constraints applied on the spurions. It turns out that the two natural possibilities allowed for the introduction of a two-by-two matrix transforming under unitary symmetries - namely, the case satisfying the reality condition used before, and the generic case - are exactly what we need.

Here, the $X_{1}$ spurion is restricted to satisfy the reality condition $X_{1}^{c}=X_{1}$, as before, whereas the $\tilde{Y}_{0}$ spurion is arbitrary in this respect. We require both spurions to satisfy the constraint of covariant constancy, as we did in section 2.2.1 The outcome is then the following: we can perform suitable $\mathrm{SU}(2)_{R_{1}}$ and $\mathrm{SU}(2)_{L_{0}}$ transformations such that the spurions reduce to three real constants and one phase

$$
\begin{aligned}
& \left.X_{1}\right|_{\text {const. }}=\xi_{1} \mathbb{1}_{2 \times 2}, \\
& \left.\tilde{Y}_{0}\right|_{\text {const. }}=\mathrm{e}^{\mathrm{i} \varphi_{Y}}\left(\begin{array}{cc}
\eta_{01} & 0 \\
0 & \eta_{02}
\end{array}\right) .
\end{aligned}
$$

The magnitude of these constants then can serve as expansion parameters, and in addition, $\tilde{Y}_{0}$, being diagonal but not proportional to the identity, allows for weak isospin breaking. Such diagonal matrices have been used in the literature ${ }^{11}$ in order to select a gauge group, but our point is that - at least for the case of reducing $\mathrm{SU}(2)$ to $\mathrm{U}(1)$ - these constant diagonal matrices can in fact be traced back to spurions and that an expansion can be built based on that. Therefore, the possibility of building a systematic expansion is guaranteed, since the appropriate terms for the renormalization procedure can be constructed with the help of the spurions 22,52. Moreover, the diagonal and constant matrices descend from fields that are considered as non-propagating at this level, pointing to a dynamical origin for this subgroup selection.

In the same gauge where the spurions are diagonalized, we get

$$
\begin{aligned}
& \left.R_{1 \mu}\right|_{\text {const. }}=g_{1} G_{1 \mu}, \\
& \left.L_{0 \mu}^{1,2}\right|_{\text {const. }}=G_{0 \mu}^{1,2}=0, \\
& \left.L_{0 \mu}^{3}\right|_{\text {const. }}=g_{0} G_{0 \mu}^{3} .
\end{aligned}
$$

\footnotetext{
10 The notations $L_{0}$ and $R_{1}$ are just names given to the groups, and are in no way related to the chirality of fermions to be introduced later.

11 See 24] among others.
}

The $R_{1 \mu}$ connection and the gauge field $g_{1} G_{1 \mu}$ are identified, whereas, due to the complex nature of the $\tilde{Y}_{0}$ spurion, only the $\mathrm{U}(1)$ subgroup of $G_{0}$ can be gauged. In this case, the identification between connections only concerns the third component: the spurion has selected the $\mathrm{U}(1)_{\tau^{3}}$ subgroup.

In order to arrive at this result, the spurions are introduced as follows: $X_{1}$ transforms as before

$$
X_{1} \longmapsto R_{1} X_{1} G_{1}^{\dagger}
$$

with $G_{1}$ the SU (2) gauge transformation, and is restricted to satisfy, as in (2.25)

$$
X_{1}^{c}=X_{1}
$$

In order to identify $G_{0}$ with the $\mathrm{U}(1)$ subgroup of SU (2), we will consider a spurion $\tilde{Y}_{0}$ which is a generic two-bytwo matrix-valued function — albeit with small entriestransforming as

$$
\tilde{Y}_{0} \longmapsto G_{0} \tilde{Y}_{0} L_{0}^{\dagger}
$$

Before we proceed to solve the constraints, we first write all leading-order terms for the unconstrained lagrangian, that is the terms of order $\mathcal{O}\left(p^{2} \epsilon^{0}\right)$. They are collected in the following lagrangian

$$
\begin{aligned}
\mathcal{L}_{\text {bosons }}^{(2,0)} & =\frac{f_{0}^{2}}{4}\left\langle D_{\mu} \Sigma_{0,1} D^{\mu} \Sigma_{0,1}^{\dagger}\right\rangle-\frac{1}{2}\left\langle G_{1 \mu \nu} G_{1}^{\mu \nu}\right\rangle \\
& -\frac{1}{2}\left\langle G_{0 \mu \nu} G_{0}^{\mu \nu}\right\rangle
\end{aligned}
$$

where the covariant derivative operator $D_{\mu}$ involves both sources $L_{0 \mu}$ and $R_{1 \mu}$, but not the dynamical gauge fields $G_{0 \mu}$ and $G_{1 \mu}$

$$
D_{\mu} \Sigma_{0,1}=\partial_{\mu} \Sigma_{0,1}-\mathrm{i} L_{0 \mu} \Sigma_{0,1}+\mathrm{i} \Sigma_{0,1} R_{1 \mu} .
$$

We now turn to the constraints: interactions with the gauge fields are introduced by requiring the spurions to satisfy the usual constraints of covariant constancy

$$
\begin{aligned}
D_{\mu} X_{1} & =0 \\
D_{\mu} \tilde{Y}_{0} & =0,
\end{aligned}
$$

where the covariant derivatives are defined in accordance with (4.10) and (4.12).

The constraint is solved as usual for the $X_{1}$ spurion, using the decomposition

$$
X_{1}=\xi_{1} U_{1}
$$

and performing an $\mathrm{SU}(2)_{R_{1}}$ transformation with

$$
R_{1}=U_{1}^{\dagger}
$$

to arrive at the expected results (4.5) and then (4.7) when the constraint is explicitly written. The SU (2) transformation $G_{1}$ remains unconstrained. 


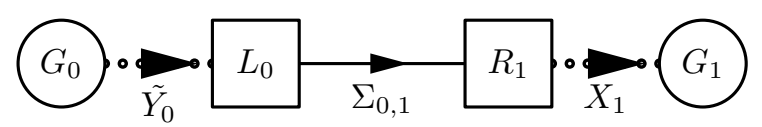

Fig. 4.1. Coupling the electroweak gauge fields to the $K=0$ moose via spurions.

As for the $\tilde{Y}_{0}$ spurion, we decompose the generic twoby-two matrix according to

$$
\tilde{Y}_{0}=\mathrm{e}^{\mathrm{i} \varphi_{Y}} G_{Y}^{\dagger} D_{Y} L_{Y},
$$

where $\varphi_{Y}$ is real and $G_{Y}, L_{Y}$ are elements of SU (2) and $D_{Y}$ is diagonal and real. Since the $\tau^{3}$ matrix commutes with $D_{Y}$, the couple $\left(G_{Y}, L_{Y}\right)$ is unique only up to a diagonal $\mathrm{U}(1)$ transformation

$$
\left(G_{Y}, L_{Y}\right) \in \frac{\mathrm{SU}(2) \times \mathrm{SU}(2)}{\mathrm{U}(1)_{\tau^{3}, \text { diagonal }}} .
$$

One can check that the number of independent real parameters in the right-hand side of (4.19) is indeed eight, as it should be. Performing the following $\mathrm{SU}(2)_{G_{0}}$ and $\mathrm{SU}(2)_{L_{0}}$ transformations

$$
\begin{aligned}
& G_{0}=\mathrm{e}^{-\mathrm{i} f \frac{\tau^{3}}{2}} G_{Y}, \\
& L_{0}=\mathrm{e}^{-\mathrm{i} f \frac{\tau^{3}}{2}} L_{Y},
\end{aligned}
$$

we get

$$
\tilde{Y}_{0}=\mathrm{e}^{\mathrm{i} \varphi_{Y}} D_{Y}
$$

independently of the gauge function $f$. Now, as a consequence of (4.16) we have

$$
D_{\mu}\left(\tilde{Y}_{0} \tilde{Y}_{0}^{\dagger}\right)=0
$$

which is explicitly written in the gauge reached by performing the transformations (4.21) and (4.22) as

$$
\partial_{\mu}\left(D_{Y}^{2}\right)-\mathrm{i} g_{0}\left[G_{0 \mu}, D_{Y}^{2}\right]=0 .
$$

From this we deduce that

$$
D_{Y}=\left(\begin{array}{cc}
\eta_{01} & 0 \\
0 & \eta_{02}
\end{array}\right)
$$

must be a constant matrix, yielding (4.6). Its two entries will be considered small, and of the same order as those of the real spurions ${ }^{12}$

$$
\eta_{01}, \eta_{02}=\mathcal{O}(\epsilon) .
$$

Furthermore, we also find that $\varphi_{Y}$ must be a constant. In addition, one concludes in the generic case where $\eta_{01} \neq$ $\eta_{02}$, that

$$
G_{0 \mu}^{1,2}=0,
$$

\footnotetext{
12 This is not forced on us by the consistency of the expansion: one could distinguish between this spurion and others, as they play a different role.
}

in this gauge. Turning to the equation (4.16) itself, we get

$$
g_{0} G_{0 \mu} D_{Y}-D_{Y} L_{0 \mu}=0 .
$$

With the help of (4.26) and (4.28), this last equation implies the promised result reproduced in details in (4.8) and (4.9).

Reaching the appropriate gauge this time involved a $G_{0}$ transformation, but it turned out that an abelian subgroup remained unconstrained: the gauge field is only restricted to lie in the third direction due to the covariant constancy conditions. Indeed, when the constraints are applied, the only degree of freedom left of the six initial real gauge functions in the $\mathrm{SU}(2)_{L_{0}} \times \mathrm{SU}(2)_{G_{0}}$ transformation is the $\mathrm{U}(1)$ transformation $f$. We may thus use the notation

$$
\left.G_{0 \mu}^{3}\right|_{\text {const. }}=b_{\mu}^{0},
$$

since the connection is an abelian one, giving the corresponding field-strength

$$
b_{\mu \nu}^{0}=\partial_{\mu} b_{\nu}^{0}-\partial_{\nu} b_{\mu}^{0} .
$$

The gauge transformation for $b_{\mu}^{0}$, is then

$$
b_{\mu}^{0} \longmapsto b_{\mu}^{0}-\frac{1}{g_{0}} \partial_{\mu} f,
$$

which is compatible with the constraints: we have a $\mathrm{U}(1)$ symmetry left. One should not be surprised to find that considering a generic spurion instead of a real one gives more restrictions on the gauge field configurations. Indeed, since we have a larger number of parameters, the covariant constancy equation implies a larger number of relations.

Upon injecting the solutions to the constraints involving the spurions, the lagrangian (4.13) becomes

$$
\begin{aligned}
\left.\mathcal{L}_{\text {bosons }}^{(2,0)}\right|_{\text {const. }} & =\frac{f_{0}^{2}}{4}\left\langle\nabla_{\mu} \Sigma_{0,1} \nabla^{\mu} \Sigma_{0,1}^{\dagger}\right\rangle-\frac{1}{2}\left\langle G_{1 \mu \nu} G_{1}^{\mu \nu}\right\rangle \\
& -\frac{1}{4} b_{\mu \nu}^{0} b^{0 \mu \nu},
\end{aligned}
$$

where the following definition applies

$$
\begin{aligned}
\nabla_{\mu} \Sigma_{0,1} & =\left.D_{\mu} \Sigma_{0,1}\right|_{\text {const. }} \\
& =\partial_{\mu} \Sigma_{0,1}-\mathrm{i} g_{0} b_{\mu}^{0} \frac{\tau^{3}}{2} \Sigma_{0,1} \\
& +\mathrm{i} g_{1} \Sigma_{0,1} G_{1 \mu} .
\end{aligned}
$$


The following terms, yielding a non-zero contribution to the $S$ parameter

$$
\begin{aligned}
\left\langle L_{0 \mu \nu} \Sigma_{0,1} R_{1}^{\mu \nu} \Sigma_{0,1}^{\dagger}\right\rangle & =\mathcal{O}\left(p^{4} \epsilon^{0}\right), \\
\left\langle L_{0 \mu \nu} \Sigma_{0,1} X_{1} G_{1}^{\mu \nu} X_{1}^{\dagger} \Sigma_{0,1}^{\dagger}\right\rangle & =\mathcal{O}\left(p^{3} \epsilon^{2}\right), \\
\left\langle G_{0 \mu \nu} \tilde{Y}_{0} \Sigma_{0,1} R_{1}^{\mu \nu} \Sigma_{0,1}^{\dagger} \tilde{Y}_{0}^{\dagger}\right\rangle & =\mathcal{O}\left(p^{3} \epsilon^{2}\right), \\
\left\langle G_{0 \mu \nu} \tilde{Y}_{0} \Sigma_{0,1} X_{1} G_{1}^{\mu \nu} X_{1}^{\dagger} \Sigma_{0,1}^{\dagger} \tilde{Y}_{0}^{\dagger}\right\rangle & =\mathcal{O}\left(p^{2} \epsilon^{4}\right),
\end{aligned}
$$

are absent at leading order: they are only corrections to the original $\mathcal{O}\left(p^{2} \epsilon^{0}\right)$ lagrangian 4.13).

Note that, whenever a term with $\tilde{Y}_{0}$ is possible, the same term with $\tilde{Y}_{0}^{c}$ can also be written down, as $\tilde{Y}_{0}^{c}$ transforms in the same manner as $\tilde{Y}_{0}$, but is independent from it for a complex spurion.

In summary, we see that the complex spurion now allows us to take into account weak isospin breaking. This is obviously what they had been introduced for in the first place: to restrict the symmetries of the model, and the space of gauge configurations. However, they turn out to be constant diagonal matrices in the gauge in which we solve the constraints. The spurions are therefore a way of introducing weak isospin breaking effects independently of the coupling constant $g_{0}{ }^{13}$ in addition to the breaking due to $g_{0} \neq 0$ itself, a distinction already mentioned in [53. In addition, the spurion formalism provides a power counting associated with these isospin breaking effects. This will be of importance when we study fermions and the mass-splittings within the doublets.

\subsection{Bosons}

At this stage, we want to show that the bosonic sector of this model is identical, at tree-level, to that of the SM with the Higgs particle removed. For this purpose, we use field redefinitions as in section 2.3 we stress again that even though these redefinitions are suitable for the discussion of the physical fields, the procedure does not involve gauge-fixing. We once again give these redefinitions as they appear after injection of the solution to the constraints applied on the spurions. The $W$ fields are defined through

$$
W_{\mu}=\frac{\mathrm{i}}{g_{1}} \Sigma_{0,1} \nabla_{\mu} \Sigma_{0,1}^{\dagger},
$$

in which case the lagrangian does not depend on the Goldstone bosons anymore: this is the consequence of the Higgs mechanism. This is sufficient for the $W^{ \pm}$fields, but we still have to work out the mixing of the neutral fields: the mass matrix in the $b_{\mu}^{0}, W_{\mu}^{3}$ basis is indeed

$$
\frac{g_{1}^{2} f_{0}^{2}}{4}\left(\begin{array}{ll}
0 & 0 \\
0 & 1
\end{array}\right)
$$

\footnotetext{
13 Though it will be argued in section 5.2 .3 that there might be cases where the power counting for spurions and for coupling constants have to be related.
}

while the kinetic term is in the same basis

$$
-\frac{1}{4}\left(\begin{array}{cc}
1+\left(\frac{g_{0}}{g_{1}}\right)^{2} & \frac{g_{0}}{g_{1}} \\
\frac{g_{0}}{g_{1}} & 1
\end{array}\right) .
$$

This is rewritten in canonical form using the definitions

$$
\begin{aligned}
W_{\mu}^{ \pm} & =\frac{\mathrm{i} \sqrt{2}}{g_{1}}\left\langle\tau^{ \pm} \Sigma_{0,1} \nabla_{\mu} \Sigma_{0,1}^{\dagger}\right\rangle, \\
A_{\mu} & =s \frac{\mathrm{i}}{g_{1}}\left\langle\tau^{3} \Sigma_{0,1} \nabla_{\mu} \Sigma_{0,1}^{\dagger}\right\rangle+\frac{1}{c} b_{\mu}^{0}, \\
Z_{\mu} & =c \frac{\mathrm{i}}{g_{1}}\left\langle\tau^{3} \Sigma_{0,1} \nabla_{\mu} \Sigma_{0,1}^{\dagger}\right\rangle,
\end{aligned}
$$

with

$$
\begin{aligned}
& c=\frac{1}{\sqrt{1+\left(\frac{g_{0}}{g_{1}}\right)^{2}}}, \\
& s=\sqrt{1-c^{2}} .
\end{aligned}
$$

The above definitions are useful in order to show that the lagrangian does not involve the Goldstone fields anymore when written with the appropriate variables. In order to relate this to the usual diagonalization in the $\mathrm{SM}$, one may rewrite these field redefinitions in the particular case where $\pi^{a}=0^{14}$ for $a=1,2,3$ in

$$
\Sigma_{0,1}=\mathrm{e}^{\mathrm{i} \frac{\pi^{a} \tau^{a}}{f_{0}}}
$$

to find

$$
\begin{aligned}
\left.A_{\mu}\right|_{\pi^{a}=0} & =c b_{\mu}^{0}+s G_{1 \mu}^{3}, \\
\left.Z_{\mu}\right|_{\pi^{a}=0} & =-s b_{\mu}^{0}+c G_{1 \mu}^{3} .
\end{aligned}
$$

One recognizes the SM tree-level formulas after performing the replacement $g_{0} \longmapsto g^{\prime}$ and $g_{1} \longmapsto g$. The value of the electric charge is as expected

$$
e=\frac{g_{0} g_{1}}{\sqrt{g_{0}^{2}+g_{1}^{2}}} .
$$

The kinetic term for the Goldstone bosons yields the following mass term

$$
\begin{aligned}
\frac{f_{0}^{2}}{4}\left\langle\nabla_{\mu} \Sigma_{0,1} \nabla^{\mu} \Sigma_{0,1}^{\dagger}\right\rangle & =M_{W}^{2}\left\langle W_{\mu}^{+} W_{\mu}^{-}\right\rangle \\
& +\frac{1}{2} M_{Z}^{2}\left\langle Z_{\mu} Z^{\mu}\right\rangle,
\end{aligned}
$$

with the SM-like definitions

$$
\begin{aligned}
M_{W}^{2} & =\frac{g_{1}^{2}}{4} f_{0}^{2}, \\
M_{Z}^{2} & =\frac{g_{1}^{2}+g_{0}^{2}}{4} f_{0}^{2} .
\end{aligned}
$$

14 To be more general, one may assume that this condition of setting the Goldstone modes to zero is achieved by gauge-fixing as one would do to define the unitary gauge in the standard manner for the SM. 


\subsection{Fermions and $U(1)_{B-L}$}

As already mentioned in (4.1), the natural symmetry of this model involves a $\mathrm{U}(1)_{B-L}$ group which is relevant for fermions. This is the subject of this section: within this minimal case, we want to describe the introduction of fermions charged under $\mathrm{U}(1)_{B-L}$. We denote the $\mathrm{U}(1)_{B-L}$ connection by $B_{\mu}$, transforming as

$$
B_{\mu} \longmapsto B_{\mu}-\partial_{\mu} \beta^{0}
$$

At this stage, $B_{\mu}$ is merely an external source (as are $L_{0 \mu}$ and $R_{1 \mu}$ ) and this is why the corresponding kinetic term does not appear in the lagrangian. The appropriate power counting is

$$
\begin{aligned}
B_{\mu} & =\mathcal{O}\left(p^{1} \epsilon^{0}\right), \\
B_{\mu \nu} & =\mathcal{O}\left(p^{2} \epsilon^{0}\right),
\end{aligned}
$$

as can be guessed from (2.11).

We will be considering elementary chiral fermion doublets $\chi_{L}$ and $\chi_{R}$, transforming with respect to the natural symmetry group $S_{\text {natural (4.1) as }}$

$$
\begin{aligned}
& \chi_{L} \longmapsto G_{1} \mathrm{e}^{-\mathrm{i} \frac{B-L}{2} \beta^{0}} \chi_{L}, \\
& \chi_{R} \longmapsto G_{0} \mathrm{e}^{-\mathrm{i} \frac{B-L}{2} \beta^{0}} \chi_{R},
\end{aligned}
$$

where we have introduced the two four-component projections $\chi_{L}, \chi_{R}$ of the Dirac spinor doublet $\chi$ which has eight complex components. The definitions are as usual

$$
\chi_{L, R}=\frac{1 \mp \gamma_{5}}{2} \chi
$$

The transformation properties of these elementary fermions, except for the $\mathrm{U}(1)$ transformation, are schematized in figure 4.2

Another possibility would be to consider fermions transforming under $L_{0}$ and $R_{1}$ instead of $G_{0}$ and $G_{1}$. Such fields would then have to be interpreted as bound states resulting from the dynamics of the techni-theory. Hence, they should be considered as composite as opposed to the elementary fermions we have just introduced above and which transform under the weak gauge groups.

Note that, as was noticed in 27,.54 for small-momentum power counting purposes, the following counting for chiral fermions has to be imposed

$$
\chi_{L}, \chi_{R}=\mathcal{O}\left(p^{1 / 2}\right) .
$$

This can be deduced for instance by requiring that the kinetic terms of these fermions appear at the same order as the kinetic terms for the bosons, thereby allowing them to be part of the effective theory as dynamical fields at the same level, or alternatively by inspection of the normalization of states. Both these conditions imply the result (4.60), with the proviso that we consider massless or light fermions, again in connection with naturalness.

We have introduced the additional $\mathrm{U}(1)_{B-L}$ symmetry, but it turns out we will be interested in the case where the corresponding gauge fields is identified with the one associated with the $\mathrm{U}(1)_{\tau^{3}}$ of section 4.1. As should be clear from what we have done up to now, we are going to impose this identification through our usual constraint of covariant constancy on a spurion. For this purpose, we introduce another non-propagating field: a complex doublet transforming as 15

$$
\phi \longmapsto G_{0} \mathrm{e}^{\mathrm{i} \frac{\beta^{0}}{2}} \phi
$$

Our choice for the $\mathrm{U}(1)$ charge of this doublet is done in order to have the proper normalization for the $B-L$ charges once the constraints are solved and the ensuing identification between $\mathrm{U}(1)$ connections is performed. The condition of covariant constancy to be imposed reads explicitly

$$
D_{\mu} \phi=\partial_{\mu} \phi-\mathrm{i}\left(g_{0} G_{0 \mu}-\frac{B_{\mu}}{2}\right) \phi=0 .
$$

We next assume that the choice of gauge described in section 4.1 has already been performed and the constraint on $\tilde{Y}_{0}$ has already been solved for. The result is then that we obtain (4.6), (4.8) and (4.9). In order to diagonalize $\tilde{Y}_{0}$, we have used a $G_{0}$ transformation as specified in (4.21). Now, solving (4.62) with our usual method of choosing the simplest gauge appears impossible: it seems a priori that the problem is overdetermined. Thanks to the constraint themselves, which restrict the gauge configurations to belong to the $\mathrm{U}(1)$ subgroup of $\mathrm{SU}(2)$, it is in fact possible to find a solution, resulting in

$$
\left.\phi\right|_{\text {const. }}=\left(\begin{array}{l}
\zeta \\
0
\end{array}\right),
$$

and

$$
\left.B_{\mu}\right|_{\text {const. }}=g_{0} G_{0 \mu}^{3}=g_{0} b_{\mu}^{0},
$$

where $\zeta$ is a real constant to which we apply for the sake of simplicity the same power counting as to the other spurions: it is counted as $\mathcal{O}(\epsilon)^{16}$. We will also see that (4.28) still holds.

To show this, we use the fact that a complex doublet can always be decomposed in terms of a real function $\zeta$ and an $\mathrm{SU}(2)$ matrix $U_{Z}$ as

$$
\phi=U_{Z}\left(\begin{array}{l}
\zeta \\
0
\end{array}\right)
$$

We then perform the following $\mathrm{SU}(2)_{G_{0}} \times \mathrm{U}(1)_{B-L}$ transformation

$$
\begin{aligned}
& G_{0}=\mathrm{e}^{-\mathrm{i} f^{\prime} \frac{\tau^{3}}{2}} U_{Z}^{\dagger}, \\
& \beta^{0}=f^{\prime},
\end{aligned}
$$

15 This complex doublet is not to be confused with the Higgs doublet of the SM, which would transform under $G_{1}$. As we shall also see in section 4.5 it does not have the same impact on fermion masses.

16 The same remark as in footnote 12 applies. 


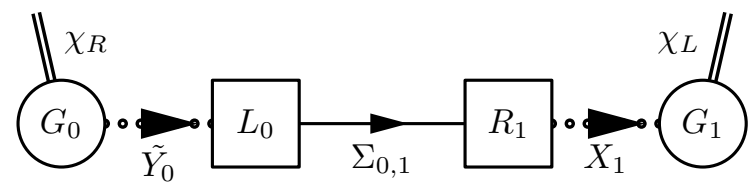

Fig. 4.2. Introduction of fermions in the model of section 4.1

with $f^{\prime}$ a gauge function to be solved for later. When this transformation is applied, $\phi$ reduces to the real function $\zeta$ in its upper component, and zero in the lower component. We then write out explicitly the components of the constraint equation (4.62) to find that $\zeta$ is indeed a constant, which is the result (4.63). We also get in this gauge

$$
\begin{gathered}
G_{0 \mu}^{\prime 1,2}=0, \\
g_{0} G_{0 \mu}^{\prime 3}=B_{\mu} .
\end{gathered}
$$

Now we see that this gauge is related to the one used in section 4.1 by

$$
G_{0 \mu}^{\prime}=V G_{0 \mu} V^{\dagger}+\frac{\mathrm{i}}{g_{0}} V \partial_{\mu} V^{\dagger},
$$

where the gauge transformation $V$ is given by

$$
V=\mathrm{e}^{-\mathrm{i} f^{\prime} \frac{\tau^{3}}{2}} U_{Z}^{\dagger} G_{Y}^{\dagger} \mathrm{e}^{\mathrm{i} f \frac{\tau^{3}}{2}} .
$$

However, since both $G_{0 \mu}^{\prime}$ and $G_{0 \mu}$ point in the third direction, the transformation $V$ only involves the exponential of a function times $\tau^{3}$ : it is a $\mathrm{U}(1)$ transformation. Therefore, by appropriately choosing $f^{\prime}$ depending on the function $f$, we can set this function to be zero, that is

$$
V=1
$$

We are then left with only one free gauge function $f$.

In summary, due to the constraints themselves, we have been able to diagonalize both spurions in the same gauge, giving

$$
G_{0 \mu}^{\prime}=G_{0 \mu} .
$$

Injecting this into (4.69), we get the desired result (4.64).

To summarize, we make a list of useful results from this section and section 4.1 we have shown that there exists a gauge in which the spurions reduce to the following constants, assumed for simplicity to be $\mathcal{O}(\epsilon)$

$$
\begin{aligned}
\left.X_{1}\right|_{\text {const. }} & =\xi_{1} \mathbb{1}_{2 \times 2}, \\
\left.\tilde{Y}_{0}\right|_{\text {const. }} & =\mathrm{e}^{\mathrm{i} \varphi_{Y}}\left(\begin{array}{cc}
\eta_{01} & 0 \\
0 & \eta_{02}
\end{array}\right), \\
\left.\phi\right|_{\text {const. }} & =\left(\begin{array}{l}
\zeta \\
0
\end{array}\right) .
\end{aligned}
$$

This result is of paramount importance since this is the standard gauge we will be using each time we want to inject the solution to the constraints in order to see the physical content of the terms. This gauge is reached by fixing nine gauge functions among the thirteen available for generic $\mathrm{SU}(2)_{G_{0}} \times \mathrm{SU}(2)_{L_{0}} \times \mathrm{SU}(2)_{R_{1}} \times \mathrm{SU}(2)_{G_{1}} \times$ $\mathrm{U}(1)_{B-L}$ transformations. We are then left with an $\mathrm{SU}(2) \times$ $\mathrm{U}(1)_{Y}$ invariance. The $\mathrm{U}(1)_{Y}$ degree of freedom is associated with the gauge function $f$. In this gauge, the following relations between connections hold

$$
\begin{aligned}
\left.R_{1 \mu}^{a}\right|_{\text {const. }} & =g_{1} G_{1 \mu}^{a}, \text { for } a=1,2,3, \\
\left.L_{0 \mu}^{1,2}\right|_{\text {const. }} & =G_{0 \mu}^{1,2}=0 \\
\left.L_{0 \mu}^{3}\right|_{\text {const. }} & =\left.g_{0} G_{0 \mu}^{3}\right|_{\text {const. }}=\left.B_{\mu}\right|_{\text {const. }} \\
& =g_{0} b_{\mu}^{0}
\end{aligned}
$$

where $G_{1 \mu}^{a}$ and $b_{\mu}^{0}$ are the only gauge fields which are nonzero. $b_{\mu}^{0}$ transforms as a $\mathrm{U}(1)$ connection

$$
b_{\mu}^{0} \longmapsto b_{\mu}^{0}-\frac{1}{g_{0}} \partial_{\mu} f
$$

Although for notational convenience we count each power of spurion amplitudes $\xi_{1}, \eta_{01}, \eta_{02}$ and $\zeta$ as $\mathcal{O}(\epsilon)$, it should be stressed once more that the final counting of various spurions can be consistently determined only taking into account loops in a full analysis of various symmetry breaking effects. This is however beyond the scope of this paper.

\subsection{Fermion couplings}

With the notations of the preceding section, we find the following possible terms involving fermions at order $\mathcal{O}\left(p^{2} \epsilon^{0}\right)$

$$
\begin{aligned}
\mathcal{L}_{\text {fermions }}^{(2,0)} & =\mathrm{i} \overline{\chi_{L}} \gamma^{\mu} D_{\mu} \chi_{L}+\mathrm{i} \overline{\chi_{R}} \gamma^{\mu} D_{\mu} \chi_{R} \\
& + \text { four-fermion interactions. }
\end{aligned}
$$

We want to show that the couplings with vector fields at lowest-order are automatically identical to those in the SM. We start from

$$
\begin{aligned}
D_{\mu} \chi_{L} & =\partial_{\mu} \chi_{L} \\
& -\mathrm{i}\left(g_{1} G_{1 \mu}+\frac{B-L}{2} B_{\mu}\right) \chi_{L}, \\
D_{\mu} \chi_{R} & =\partial_{\mu} \chi_{R} \\
& -\mathrm{i}\left(g_{0} G_{0 \mu}+\frac{B-L}{2} B_{\mu}\right) \chi_{R},
\end{aligned}
$$


which become upon application of the constraints and in the standard gauge

$$
\begin{aligned}
\nabla_{\mu} \chi_{L} & =\left.D_{\mu} \chi_{L}\right|_{\text {const. }} \\
& =\partial_{\mu} \chi_{L} \\
& -\mathrm{i}\left(g_{1} G_{1 \mu}+g_{0} \frac{B-L}{2} b_{\mu}^{0}\right) \chi_{L} \\
\nabla_{\mu} \chi_{R} & =\left.D_{\mu} \chi_{R}\right|_{\text {const. }} \\
& =\partial_{\mu} \chi_{R} \\
& -\mathrm{i} g_{0} b_{\mu}^{0}\left(\frac{\tau^{3}}{2}+\frac{B-L}{2}\right) \chi_{R} .
\end{aligned}
$$

Using unitary rotations involving the SU (2) matrix-valued field $U_{1}$ as defined in (4.17), we next perform the following fields redefinitions for fermions

$$
\begin{aligned}
\psi_{L} & =\Sigma_{0,1} U_{1} \chi_{L}, \\
\psi_{R} & =\chi_{R},
\end{aligned}
$$

which are inoffensive from the point of view of anomalies as long as we have three quarks for one lepton, since the trace of $B-L$ over fermions is then equal to zero. Denoting

$$
\psi=\psi_{L}+\psi_{R}
$$

and using the field redefinitions we have introduced for vector fields in (4.42 4.44), we find that (4.81) becomes upon application of the constraints in the standard gauge

$$
\begin{aligned}
\left.\mathcal{L}_{\text {fermions }}^{(2,0)}\right|_{\text {const. }}= & i \bar{\psi} \gamma^{\mu} \partial_{\mu} \psi+e \bar{\psi} \gamma^{\mu} Q \psi A_{\mu} \\
& +\frac{e}{c s} \bar{\psi} \gamma^{\mu}\left\{\frac{\tau^{3}}{2} \frac{\left(1-\gamma_{5}\right)}{2}-s^{2} Q\right\} \psi Z_{\mu} \\
& +\frac{1}{\sqrt{2}} \frac{e}{s} \bar{\psi} \gamma^{\mu} \tau^{\mp} \frac{\left(1-\gamma_{5}\right)}{2} \psi W_{\mu}^{ \pm},
\end{aligned}
$$

with

$$
Q=\frac{\tau^{3}}{2}+\frac{B-L}{2} .
$$

This is the desired SM-like result: anomalous couplings would come from terms such as

$$
\begin{aligned}
& \mathrm{i} \overline{\chi_{L}} \gamma^{\mu} X_{1}^{\dagger} \Sigma_{0,1}^{\dagger}\left(D_{\mu} \Sigma_{0,1}\right) X_{1} \chi_{L}=\mathcal{O}\left(p^{2} \epsilon^{2}\right), \\
& \mathrm{i} \overline{\chi_{R}} \gamma^{\mu} \tilde{Y}_{0} \Sigma_{0,1}\left(D_{\mu} \Sigma_{0,1}^{\dagger}\right) \tilde{Y}_{0}^{\dagger} \chi_{R}=\mathcal{O}\left(p^{2} \epsilon^{2}\right),
\end{aligned}
$$

and are therefore of higher order in the spurion expansion. This is automatically obtained in our spurion formalism without any additional assumptions and it represents a new result compared to the literature 48,50,51,49,53. It should be stressed that such terms would result in a violation of the universality of left-handed couplings, and would also allow couplings of the right-handed fermions to the $W^{ \pm}$. It is therefore very satisfactory to find that they are automatically suppressed as a by-product of our spurion formalism, which we have introduced in relation with other considerations pertaining to naturalness, and which in addition allows us to describe the breaking of weak isospin.

Anomalous magnetic moment terms will also be suppressed in the spurion expansion, and are suppressed by an unknown dimensionful scale, as are the four-fermion interactions.

Since the lowest-order couplings of the fermions are as in the SM and since we have recovered the same treelevel relations as in the SM for the bosonic sector - in particular, one may notice that the custodial symmetry [55] is implemented by the $\mathrm{SU}(2)_{L_{0}}$ group - it should then be no surprise that an explicit calculation gives zero treelevel values for the $S, T, U$ parameters [8] in this model.

\subsection{Fermion masses}

In order to construct fermion mass terms invariant under the whole symmetry $S_{\text {natural, }}$ and given the transformation properties of the fermions (4.57) and (4.58), spurions are necessary. In this section, we concentrate on the lowest-order terms $\mathcal{O}\left(p^{1} \epsilon^{2}\right)$, i.e. quadratic forms without derivatives, with two spurion insertions. One may conceive a joint power counting for momenta and spurions such that the kinetic term $\mathcal{O}\left(p^{2} \epsilon^{0}\right)$ and the mass term $\mathcal{O}\left(p^{1} \epsilon^{2}\right)$ would appear at the same order. We first consider quarks, and use $i, j$ as generation indices. The most general $\mathcal{O}\left(p^{1} \epsilon^{2}\right)$ mass term reads

$$
\begin{aligned}
\mathcal{L}_{\mathrm{quarks}}^{(1,2)} & =-m_{1 i j} \bar{\chi}_{L} X_{1}^{\dagger} \Sigma_{0,1}^{\dagger} \tilde{Y}_{0}^{\dagger} \chi_{R j} \\
& -m_{1 i j}^{*} \bar{\chi}_{R} \tilde{Y}_{0} \Sigma_{0,1} X_{1} \chi_{L i} \\
& -m_{2 i j} \bar{\chi}_{L} X_{1}^{\dagger} \Sigma_{0,1}^{\dagger} \tilde{Y}_{0}^{c \dagger} \chi_{R j} \\
& -m_{2 i j}^{*} \bar{\chi}_{R} \tilde{Y}_{0}^{c} \Sigma_{0,1} X_{1} \chi_{L i} .
\end{aligned}
$$

Using the notations

$$
\begin{aligned}
m_{i j}^{u} & =\xi_{1}\left(\eta_{01} \mathrm{e}^{-\mathrm{i} \varphi_{Y}} m_{1 i j}+\eta_{02} \mathrm{e}^{\mathrm{i} \varphi_{Y}} m_{2 i j}\right) \\
m_{i j}^{d} & =\xi_{1}\left(\eta_{02} \mathrm{e}^{-\mathrm{i} \varphi_{Y}} m_{1 i j}+\eta_{01} \mathrm{e}^{\mathrm{i} \varphi_{Y}} m_{2 i j}\right) \\
\psi_{i} & \longrightarrow\left(\begin{array}{c}
u_{i} \\
d_{i}
\end{array}\right)
\end{aligned}
$$

equation (4.93) becomes, when the constraints are solved

$$
\begin{aligned}
\left.\mathcal{L}_{\text {quarks }}^{(1,2)}\right|_{\text {const. }} & =-m_{i j}^{u} \overline{u_{L}} u_{R j}-m_{i j}^{u *} \overline{u_{R}}{ }_{j} u_{L i} \\
& -m_{i j}^{d} \overline{d_{L i}} d_{R j}-m_{i j}^{d *} \overline{d_{R j}} d_{L i} .
\end{aligned}
$$

In the generic power counting, both the masses and the mass-splittings within doublets will be counted as $\mathcal{O}\left(p^{0} \epsilon^{2}\right)$. From the notation in (4.97) we see that the masses and splittings for different doublets are independent of each other, allowing us to account for the masses of the fermions in a satisfactory way without the presence of physical scalars in the spectrum. We remark that the freedom is the same as in the SM, concerning both the magnitude and phases of the coefficients: the additional parameters $\xi_{1}, \eta_{01}$ and $\eta_{02}$ set the scale of the masses, whereas the phase $\varphi_{Y}$ 
can be absorbed in a redefinition of the matrices $m_{1}$ and $m_{2}$. Despite the fact that here the origin of the fermion masses is not the standard Higgs mechanism, the pattern of CKM mixing and $C P$ violation is identical to the SM: no predictivity is lost or gained at this level.

In the lepton sector, we can also write down terms analogous to those of equation (4.93). In addition, one can construct new operators involving the leptons and which are completely invariant under $S_{\text {natural }}$. These are the first terms we encounter involving the spurion $\phi$

$$
\begin{aligned}
& N_{R i}=\phi^{\dagger} \chi_{R i}=\mathcal{O}\left(p^{1 / 2} \epsilon^{1}\right), \\
& N_{L i}=\phi^{\dagger} \tilde{Y}_{0} \Sigma_{0,1} X_{1} \chi_{L i}=\mathcal{O}\left(p^{1 / 2} \epsilon^{3}\right),
\end{aligned}
$$

and a similar term with the replacement $\tilde{Y}_{0} \longmapsto \tilde{Y}_{0}^{c}$. We have given the simplest power counting for these operators, assuming a common power counting for all the spurions. However, the fact that the spurion description is common to all the symmetry-reducing mechanisms does not imply that the spurions must have the same power counting. In particular, one may entertain the view that the $\phi$ spurion should be counted at a different level. In the remainder of this section, we will therefore give the powers of $\zeta, \xi_{1}$ and $\eta_{0}$ (where we have generically used $\eta_{0}$ for both $\eta_{01}$ and $\eta_{02}$ ) which appear in the operators, instead of the powers of $\epsilon$.

With the $N_{L, R}$ operators, we can write down Lorentzinvariant terms violating lepton number. We recall that, up to now, we have not encountered any interactions of the right-handed neutrinos. To elucidate the physical content of the new terms involving $N_{R}$ and $N_{L}$, we use the following notation for the lepton sector

$$
\psi_{i} \longrightarrow\left(\begin{array}{c}
\nu_{i} \\
e_{i}
\end{array}\right) .
$$

Using the definitions (4.86) and (4.87) for the fermion fields in the unitary gauge and solving the constraints then leads to

$$
\begin{aligned}
& \left.N_{R i}\right|_{\text {const. }}=\zeta \nu_{R i}, \\
& \left.N_{L i}\right|_{\text {const. }}=\mathrm{e}^{\mathrm{i} \varphi_{Y}} \zeta \xi_{1} \eta_{01} \nu_{L i} .
\end{aligned}
$$

These operators project out the neutrino components of the doublet, up to multiplications by constant factors. From (4.101) and (4.102), we deduce that the right-handed Majorana masses will be proportional to $\zeta^{2}$, while the lefthanded Majorana masses will contain factors of $\zeta^{2} \xi_{1}^{2} \eta_{0}^{2}$. We can also construct additional Dirac mass-terms of order $\zeta^{2} \xi_{1} \eta_{0}$ for neutrinos, whereas the ones analogous to the mass matrix for the quarks (4.93) are proportional to $\xi_{1} \eta_{0}$. All the new terms are absent in the case of quarks due to non-invariance with respect to $\mathrm{U}(1)_{B-L}$ : only for leptons do they respect the full $S_{\text {natural }}$ symmetry, opening the possibility for lepton number violation driven by the neutrino sector. This would in fact be a place to evaluate the orders of magnitude of the various spurions when confronted to data on lepton flavor violation; however, we will leave this question open at this stage.
In addition to this, we have learned how the $\phi$ spurion is related to naturalness: it introduces lepton number violation. This is indeed the only physical consequence of the $\phi$ spurion at this level.

To summarize, we have seen that the minimal model for EWSB, which involves the dynamical Higgs mechanism, and no physical Higgs boson, fits quite nicely in our framework: the $\mathrm{U}(1)_{Y}$ gauge group is introduced naturally within the spurion formalism, which gives us a possibility to properly account for the masses of the fermions while suppressing non-standard terms. With this firmer basis for the neglect of unwanted terms, we may then embark on a more thorough study of the effective theory expansion for this minimal case, along the lines of [54. This is however outside the scope of this paper.

\section{Two examples of non-minimal Higgs-less models}

The fact that all particles that are considered light must be included in our effective lagrangian implies that there is no single effective lagrangian once one allows for new particles not too far above the weak scale: we will give examples of such cases in this section. In fact we will first study in this section a case were there are vector resonances in the low-energy spectrum in addition to the minimal particle content, and then one where there is a triplet of PGBs: barring supersymmetry, the only known way to protect physical scalar masses from radiative corrections is for them to be PGBs. This last scenario still deserves the name of Higgs-less model, as these scalars are not related to the SM Higgs.

\subsection{Case with $W^{\prime}$ and $Z^{\prime}$}

The previous section was a showcase to introduce our method of using linear mooses equipped with spurions as toy-models of EWSB. We now want to give some insight into the next simplest case which involves an additional gauge multiplet $(K=1)$. This model will then include excited vectors $W^{\prime}$ and $Z^{\prime}$. The idea is to discuss the simplest possibility that gives corrections to the SM-like relations we have seen in section 4 corrections appear in this case at tree-level due to these excited vectors, which are assumed to be heavier than the $W^{ \pm}$and $Z$, but still lighter than the next resonances. The generalization to models with more and more excited vectors is always possible, and may be motivated by considerations of tree-level unitarity [34 35], which we will not get into here.

Our model is depicted in Fig. 5.1 with the elementary fermions already included. The transformation properties of the fermion doublets are thus the following

$$
\begin{aligned}
\chi_{L} \longmapsto G_{2} \mathrm{e}^{-\mathrm{i} \frac{B-L}{2} \beta^{0}} \chi_{L}, \\
\chi_{R} \longmapsto G_{0} \mathrm{e}^{-\mathrm{i} \frac{B-L}{2} \beta^{0}} \chi_{R},
\end{aligned}
$$

and the definitions are as before for all the fields. 


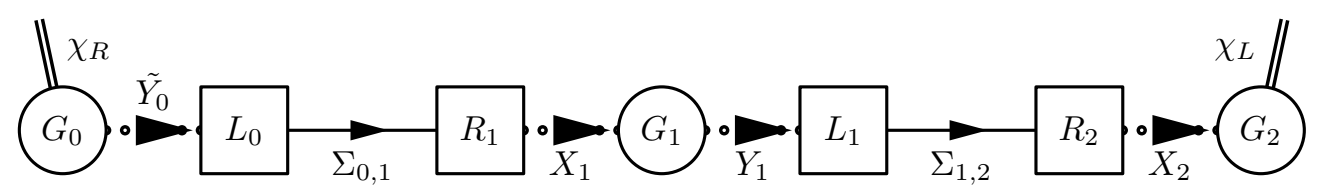

Fig. 5.1. A model for EWSB with one internal site.

We will adopt the view that the internal gauge group, which has a particular status since it describes the lowenergy sector of the techni-theory, can be assumed to have a stronger gauge coupling than the two weak gauge fields without incoherences

$$
\frac{g_{0}}{g_{1}}, \frac{g_{2}}{g_{1}} \ll 1
$$

We will then give the final results as an expansion in $1 / g_{1}$ so that it is clear how we recover the limit of section 4 when $g_{1}$ goes to infinity, as one would expect. Our model is akin to that of [50,56], but without reference to the 'hidden symmetry' assumption [57]. Furthermore, our spurion formalism enables us to describe the fermion sector (masses and couplings to vector bosons) without additional assumptions: indeed, we have seen in section 4 that anomalous couplings are suppressed by powers of $\epsilon$, and this result will still hold here.

We focus for simplification on the case where $f_{1}=f_{0}=$ $f$ at tree-level, in order to avoid unreadable formulas.

The natural symmetry of this model is

$$
S_{\text {natural }}=\mathrm{SU}(2)^{7} \times \mathrm{U}(1)_{B-L},
$$

which gets reduced via the constraints on the spurions, to

$$
S_{\text {reduced }}=\mathrm{SU}(2)^{2} \times \mathrm{U}(1)_{Y} .
$$

\subsubsection{Physical fields}

From the above definitions, we find that the $\mathcal{O}\left(p^{2} \epsilon^{0}\right)$ lagrangian is

$$
\begin{aligned}
\mathcal{L}^{(2,0)} & =\frac{f^{2}}{4}\left\langle D_{\mu} \Sigma_{0,1} D^{\mu} \Sigma_{0,1}^{\dagger}\right\rangle+\frac{f^{2}}{4}\left\langle D_{\mu} \Sigma_{1,2} D^{\mu} \Sigma_{1,2}^{\dagger}\right\rangle \\
& -\frac{1}{2}\left\langle G_{0 \mu \nu} G_{0}^{\mu \nu}\right\rangle-\frac{1}{2}\left\langle G_{1 \mu \nu} G_{1}^{\mu \nu}\right\rangle-\frac{1}{2}\left\langle G_{2 \mu \nu} G_{2}^{\mu \nu}\right\rangle \\
& +\mathrm{i} \overline{\chi_{R}} \gamma^{\mu} D_{\mu} \chi_{L}+\mathrm{i} \overline{\chi_{R}} \gamma^{\mu} D_{\mu} \chi_{R} \\
& + \text { four-fermion interactions. }
\end{aligned}
$$

The solution to the constraints

$$
\begin{aligned}
D_{\mu} X_{1} & =0, \\
D_{\mu} Y_{1} & =0,
\end{aligned}
$$

is as in section [2.2.1. Solving the constraints

$$
\begin{aligned}
D_{\mu} X_{2} & =0, \\
D_{\mu} \tilde{Y}_{0} & =0, \\
D_{\mu} \phi & =0,
\end{aligned}
$$

where

$$
\phi \longmapsto G_{0} \mathrm{e}^{\mathrm{i} \frac{\beta^{0}}{2}} \phi
$$

as before, proceeds in analogy with sections 4.1 and 4.3 The lowest-order mass term for quarks is of order $\mathcal{O}\left(p^{1} \epsilon^{4}\right)$

$$
\begin{aligned}
\mathcal{L}_{\text {quarks }}^{(1,4)} & =-m_{1 i j} \bar{\chi}_{L i} X_{2}^{\dagger} \Sigma_{1,2}^{\dagger} Y_{1}^{\dagger} X_{1}^{\dagger} \Sigma_{0,1}^{\dagger} \tilde{Y}_{0}^{\dagger} \chi_{R j} \\
& -m_{1 i j}^{*} \bar{\chi}_{j} \tilde{Y}_{0} \Sigma_{0,1} X_{1} Y_{1} \Sigma_{1,2} X_{2} \chi_{L i} \\
& -m_{2 i j} \bar{\chi}_{i} X_{2}^{\dagger} \Sigma_{1,2}^{\dagger} Y_{1}^{\dagger} X_{1}^{\dagger} \Sigma_{0,1}^{\dagger} \tilde{Y}_{0}^{c \dagger} \chi_{R j} \\
& -m_{2 i j}^{*} \bar{\chi}_{j} \tilde{Y}_{0}^{c} \Sigma_{0,1} X_{1} Y_{1} \Sigma_{1,2} X_{2} \chi_{L i} .
\end{aligned}
$$

The masses and splittings are thus of order $\mathcal{O}\left(p^{0} \epsilon^{4}\right)$ : in contrast with the result $\mathcal{O}\left(p^{0} \epsilon^{2}\right)$ we have found in the minimal case, cf. section 4.5 The power counting for spurions actually depends on the total number of spurions involved along the chain, i.e. on the length $K$ of the moose. This has to be taken into account when comparing the power counting between $\epsilon$ and $p$ on physical grounds.

Performing the proper field redefinitions, we find that this model yields $W^{ \pm}$and $Z^{0}$ vector bosons as well as a massless photon. There are in addition $W^{\prime \pm}$ and $Z^{\prime}$ resonances, which also couple directly to fermions. We will resort to the formalism of oblique parameters in order to make contact with the literature, however, we will use the definitions of these parameters in terms of observables 58 . [59] as opposed to two-point functions. The field redefinitions are as follows - once again, as they appear after solving the constraints on the spurions-

$$
\begin{aligned}
& g_{1} W_{1 \mu}=\mathrm{i} \Sigma_{0,1} \nabla_{\mu} \Sigma_{0,1}^{\dagger}, \\
& g_{2} W_{2 \mu}=\mathrm{i} \Sigma_{0,1} \Sigma_{1,2} \nabla_{\mu}\left(\Sigma_{1,2}^{\dagger} \Sigma_{0,1}^{\dagger}\right) .
\end{aligned}
$$

We then use an orthogonal transformation for the charged fields

$$
\left(\begin{array}{c}
W_{\mu}^{ \pm} \\
W_{\mu}^{\prime \pm}
\end{array}\right)=\left(\begin{array}{cc}
\cos \gamma & -\sin \gamma \\
\sin \gamma & \cos \gamma
\end{array}\right)\left(\begin{array}{l}
W_{1 \mu}^{ \pm} \\
W_{2 \mu}^{ \pm}
\end{array}\right)
$$

where $\cos \gamma$ is found to be

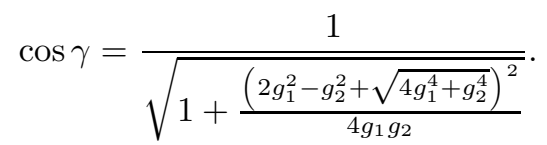

The matrix $N$ for the neutral fields involves rescalings, and is defined as

$$
\left(\begin{array}{c}
b_{\mu}^{0} \\
W_{2 \mu} \\
W_{1 \mu}
\end{array}\right)=N\left(\begin{array}{c}
A_{\mu} \\
Z_{\mu} \\
Z_{\mu}^{\prime}
\end{array}\right)
$$


The entries of this matrix reappear constantly in calculations, but their full expressions are rather lengthy, so we will not write out the $N_{i j}$ 's explicitly in the equations, but rather perform the expansion in $1 / g_{1}$. One finds the following tree-level expressions for the masses of the $W^{ \pm}$ and $Z^{0}$

$$
\begin{aligned}
M_{W}^{2} & =\frac{g_{2}^{2}}{8} f^{2}-\frac{1}{32} \frac{g_{2}^{4}}{g_{1}^{2}} f^{2}+\mathcal{O}\left(\frac{1}{g_{1}^{4}}\right), \\
M_{Z}^{2} & =\frac{g_{2}^{2}+g_{0}^{2}}{8} f^{2} \\
& -\frac{1}{32} \frac{\left(g_{0}^{2}-g_{2}^{2}\right)^{2}}{g_{1}^{2}} f^{2}+\mathcal{O}\left(\frac{1}{g_{1}^{4}}\right),
\end{aligned}
$$

and for the masses of the $W^{\prime \pm}$ and $Z^{\prime}$

$$
\begin{aligned}
& M_{W^{\prime}}^{2}=\frac{g_{1}^{2}}{2} f^{2}+\frac{g_{2}^{2}}{8} f^{2}+\mathcal{O}\left(\frac{1}{g_{1}^{2}}\right), \\
& M_{Z^{\prime}}^{2}=\frac{g_{1}^{2}}{2} f^{2}+\frac{g_{2}^{2}+g_{0}^{2}}{8} f^{2}+\mathcal{O}\left(\frac{1}{g_{1}^{2}}\right) .
\end{aligned}
$$

\subsubsection{Remarks}

The electric charge is given by

$$
e=\frac{g_{0} g_{2}}{\sqrt{g_{0}^{2}+g_{2}^{2}}}-\frac{1}{2 g_{1}^{2}} \frac{g_{0}^{3} g_{2}^{3}}{\left(g_{2}^{2}+g_{0}^{2}\right)^{\frac{3}{2}}}+\mathcal{O}\left(\frac{1}{g_{1}^{4}}\right),
$$

so that in this case, we are not in a position to make contact with the SM by a simple replacement as in section 4 but we need to study observables and check whether they obey the same relations as in the SM, or whether there are corrections relative to the SM at leading order. Details about the determination of the oblique parameters are given in appendix $\AA$ for illustration purposes. We only discuss here the $S$ parameter at tree-level. We get

$$
\alpha S=\frac{1}{g_{1}^{2}} \frac{g_{0}^{2} g_{2}^{2}}{g_{2}^{2}+g_{0}^{2}}+\mathcal{O}\left(\frac{1}{g_{1}^{4}}\right) .
$$

The sign and magnitude of this contribution is as expected from the result (3.14), using the relation [6] which gives the value of $S$ in the electroweak model once the value of $L_{10}$ for the corresponding non-linear sigma model (obtained from the moose after integration of the resonances) is known

$$
S=-16 \pi L_{10}
$$

Of course, the $S$ parameter would have been further suppressed had we chosen $f_{1} \ll f_{0}$ as already mentioned in section 3.2 Indeed, the suppression by powers of ratios of coupling-constants cannot be taken too far as it implies that $g_{1}$ is not small, and is thus threatening the consistency of the loop expansion as well as casting a doubt on the meaning of the power counting for $g_{1}$. We emphasize that this is a weak point of the formulation even though in practice, we shall not be dealing with the loop expansion for this model.
We further point out that the decoupling of the $W^{\prime}$ and $Z^{\prime}$ for tree-level relations is achieved in the limit $g_{1} \longrightarrow$ $+\infty$, as can be deduced from the formulas of appendix A in which case one obtains a shorter moose - that of section 4 . One may wish to consider the general case with additional resonances, but we will not pursue further in that direction as far as this paper is concerned.

As a conclusion to this section, we stress the fact that we have limited ourselves to the tree-level in the effective theory formalism. This formalism is to be carried out as a loop expansion involving renormalization of counter-terms of higher and higher orders: there are thus more and more constants to determine each time we ask for more precision. As long as there is no way to estimate the values of these constants at a given scale ${ }^{17}$, other than experiment, it is difficult to say whether or not there really is a conflict with experiment. Here we have included the first excited vector states in our effective lagrangian, which in practice means that we assume their effects to be larger than those of loops. We believe that this is a possibility, but the reader should be aware of the fact that limiting oneself to tree diagrams while at the same time considering the limit (5.3) is not obviously safe. Note that the considerations following (2.58) should be borne in mind as well. Given these limitations, we do not push the phenomenological discussion of this model further, but instead, turn to another possibility: that of having PGBs as the first additional states in the theory.

\subsection{Case with a triplet of PGBs}

We now describe the case where the linear moose is extended by connecting the two ends via spurions and a gauge group. Since we are interested in applications to the electroweak sector, this additional gauge group should only be the U(1) subgroup of $\mathrm{SU}(2)$, which is selected by the spurions. We will consider the simplest case, that with one internal site $(K=1)$ as shown in figure 5.2 The effective theory for this model is constructed along our standard line of reasoning, and we will find that the spectrum consists in the $W^{ \pm}, Z^{0}$, the photon, and a triplet of pseudo-scalars. Note that, as was done in 5.1 one may consider a longer chain with additional gauge groups with larger gauge couplings, yielding $W^{\prime}$ and $Z^{\prime}$ resonances: the discussion of the $S, T, U$ parameters would then be similar.

The natural symmetry of the model is in this case

$$
S_{\text {natural }}=\mathrm{SU}(2)^{6} \times \mathrm{U}(1)_{B-L},
$$

while the reduced symmetry, obtained after applying the constraints, is

$$
S_{\text {reduced }}=\mathrm{SU}(2) \times \mathrm{U}(1)_{Y} .
$$

17 Some authors have recently revived the idea that there may be a way to perform calculations for models of EWSB with strong dynamics, using the correspondence between this case and the five-dimensional models with warped geometry where the gauge symmetries get broken on branes $60 \begin{array}{ll}61 & 61\end{array}$. 


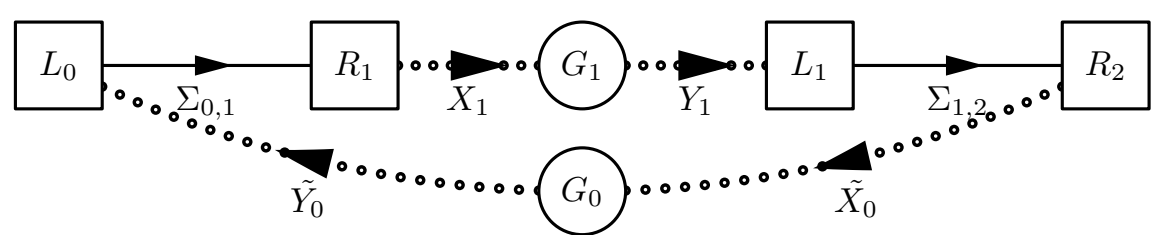

Fig. 5.2. The simplest moose model for EWSB with scalars in the spectrum.

\subsubsection{Complex spurions in a closed moose}

We introduce the spurions $\tilde{Y}_{0}, \tilde{X}_{0}, \phi$ with the following transformation properties

$$
\begin{aligned}
\tilde{Y}_{0} & \longmapsto G_{0} \tilde{Y}_{0} L_{0}^{\dagger}, \\
\phi & \longmapsto G_{0} \mathrm{e}^{\mathrm{i} \frac{\beta^{0}}{2}} \phi, \\
\tilde{X}_{0} & \longmapsto R_{2} \tilde{X}_{0} G_{0}^{\dagger},
\end{aligned}
$$

where $\tilde{Y}_{0}, \tilde{X}_{0}$ are two-by-two complex matrices and $\phi$ is a complex doublet. At this stage, the complex doublet $\phi$ is not necessary as we have not yet introduced fermions. Still, we want to show how the constraints on these three spurions are solved at the same time: we have seen in section 4.3 how the constraints

$$
\begin{aligned}
D_{\mu} \tilde{Y}_{0} & =0, \\
D_{\mu} \phi & =0,
\end{aligned}
$$

could be solved simultaneously, resulting in

$$
\begin{aligned}
\left.G_{0 \mu}^{1,2}\right|_{\text {const. }} & =0 \\
\left.L_{0 \mu}^{1,2}\right|_{\text {const. }} & =0 \\
\left.L_{0 \mu}^{3}\right|_{\text {const. }} & =\left.B_{\mu}\right|_{\text {const. }}=\left.g_{0} G_{0 \mu}^{3}\right|_{\text {const. }} \\
& =g_{0} b_{\mu}^{0},
\end{aligned}
$$

after an appropriate choice of gauge. Solving for both spurion constraints (5.31) and (5.32) using the same $G_{0}$ transformation was possible thanks to to the restrictions implied by the constraints themselves: a $\mathrm{U}(1)$ transformation was all that was required to connect the two configurations, and we had enough freedom to choose the $f^{\prime}$ gauge function for our purposes. We now want to solve in addition the constraint on the $\tilde{X}_{0}$ spurion.

Let us recall that both spurions $\tilde{Y}_{0}, \tilde{X}_{0}$ are assumed to be generic two-by-two matrices, except that their entries must be functions with small modulus, to be counted as order $\epsilon$. Using the same procedure as in section 4.3 we shall see that one can solve the constraint to be applied on $\tilde{X}_{0}$

$$
D_{\mu} \tilde{X}_{0}=0
$$

in the same gauge. The result will turn out to be the expected one: the spurion is constant and can be written

$$
\left.\tilde{X}_{0}\right|_{\text {const. }}=\mathrm{e}^{\mathrm{i} \varphi_{X}}\left(\begin{array}{cc}
\xi_{01} & 0 \\
0 & \xi_{02}
\end{array}\right),
$$

and the connections are identified, giving us the following

$$
\begin{aligned}
& \left.R_{2 \mu}^{1,2}\right|_{\text {const. }}=0, \\
& \left.R_{2 \mu}^{3}\right|_{\text {const. }}=g_{0} G_{0 \mu}^{3},
\end{aligned}
$$

while we also recover (5.33).

In order to show this, we assume that the constraint (5.31) and (5.32) on $\tilde{Y}_{0}$ and $\phi$ have been solved as in sections 4.1 and 4.3 respectively. This involves the gauge function $f^{\prime}$ of (4.66) and (4.67), which is now fixed with respect to $f$. We use the following decomposition for $\tilde{X}_{0}$

$$
\tilde{X}_{0}=\mathrm{e}^{\mathrm{i} \varphi_{X}} R_{X}^{\dagger} D_{X} G_{X}
$$

where $\varphi_{X}$ is real, $R_{X}, G_{X}$ are elements of SU (2) and $D_{X}$ is diagonal and real. The same remark concerning the number of parameters as made after (4.19) applies. We then perform the following $\mathrm{SU}(2)_{G_{0}}$ and $\mathrm{SU}(2)_{R_{2}}$ transformations

$$
\begin{aligned}
& G_{0}=\mathrm{e}^{-\mathrm{i} f^{\prime \prime} \frac{\tau^{3}}{2}} G_{X}, \\
& R_{2}=\mathrm{e}^{-\mathrm{i} f^{\prime \prime} \frac{\tau^{3}}{2}} R_{X} .
\end{aligned}
$$

We have again introduced a gauge function $f^{\prime \prime}$, to be solved for later. In this gauge, we have

$$
\tilde{X}_{0}=\mathrm{e}^{\mathrm{i} \varphi_{X}} D_{X}
$$

independently of our choice for $f^{\prime \prime}$, due to the remark following (4.19). We can then derive, along the same lines as for $\tilde{Y}_{0}$, that $D_{X}$

$$
D_{X}=\left(\begin{array}{cc}
\xi_{01} & 0 \\
0 & \xi_{02}
\end{array}\right)
$$

is a constant matrix. As usual, we impose the following power counting for the two constants appearing in this matrix

$$
\xi_{01}, \xi_{02}=\mathcal{O}(\epsilon)
$$

In the gauge specified by (5.41) and (5.42), we find, writing the covariant constancy equation component by component

$$
G_{0 \mu}^{\prime \prime 1,2}=0 .
$$

However $G_{0 \mu}^{\prime \prime}$ is related via

$$
G_{0 \mu}^{\prime \prime}=W G_{0 \mu} W^{\dagger}+\frac{\mathrm{i}}{g_{0}} W \partial_{\mu} W^{\dagger},
$$


with the gauge transformation $W \in \mathrm{SU}(2)$, to the connection $G_{0 \mu}$ we have found when solving the constraints for $\tilde{Y}_{0}$ and $\phi$. The expression for $W$ indeed reads

$$
W=\mathrm{e}^{-\mathrm{i} f^{\prime \prime} \frac{\tau^{3}}{2}} G_{X} G_{Y}^{\dagger} \mathrm{e}^{\mathrm{i} f \frac{\tau^{3}}{2}} .
$$

However, since both $G_{0 \mu}$ and $G_{0 \mu}^{\prime \prime}$ point in the third direction, we can deduce that $W$ only involves a $\mathrm{U}(1)_{\tau^{3}}$ transformation. Therefore, we can choose the function $f^{\prime \prime}$ so as to have

$$
W=1 .
$$

Thus, due to the constraints (5.36) and (5.31), we have been able to diagonalize all three spurions in the same gauge, giving

$$
G_{0 \mu}^{\prime \prime}=G_{0 \mu}^{\prime}=G_{0 \mu}
$$

We also find that $\varphi_{X}$ is a constant, and that

$$
R_{2 \mu}^{a}=g_{0} G_{0 \mu}^{a}, \text { for } a=1,2,3 .
$$

In summary, we have found a gauge in which the spurions are rewritten as the following constant matrices

$$
\begin{aligned}
\left.\tilde{X}_{0}\right|_{\text {const. }} & =\mathrm{e}^{\mathrm{i} \varphi_{X}}\left(\begin{array}{cc}
\xi_{01} & 0 \\
0 & \xi_{02}
\end{array}\right), \\
\left.\tilde{Y}_{0}\right|_{\text {const. }} & =\mathrm{e}^{\mathrm{i} \varphi_{Y}}\left(\begin{array}{cc}
\eta_{01} & 0 \\
0 & \eta_{02}
\end{array}\right), \\
\left.\phi\right|_{\text {const. }} & =\left(\begin{array}{l}
\zeta \\
0
\end{array}\right) .
\end{aligned}
$$

due to the constraints. In addition, the following connections are identified

$$
\begin{aligned}
\left.R_{2 \mu}^{1,2}\right|_{\text {const. }} & =\left.L_{0 \mu}^{1,2}\right|_{\text {const. }}=\left.g_{0} G_{0 \mu}^{1,2}\right|_{\text {const. }} \\
& =0, \\
\left.R_{2 \mu}^{3}\right|_{\text {const. }} & =\left.L_{0 \mu}^{3}\right|_{\text {const. }}=\left.B_{\mu}\right|_{\text {const. }}=\left.g_{0} G_{0 \mu}^{3}\right|_{\text {const. }} \\
& =g_{0} b_{\mu}^{0},
\end{aligned}
$$

This proves the result announced above. Again, the only invariance left from the original $\mathrm{SU}(2)_{L_{0}} \times \mathrm{SU}(2)_{G_{0}} \times$ $\mathrm{U}(1)_{B-L}$ is the $\mathrm{U}(1)_{Y}$ degree of freedom, under which

$$
b_{\mu}^{0} \longmapsto b_{\mu}^{0}-\frac{1}{g_{0}} \partial_{\mu} f
$$

with the identification (5.56).

As for the constraints on the real spurions $X_{1}, Y_{1}$

$$
\begin{aligned}
D_{\mu} X_{1} & =0, \\
D_{\mu} Y_{1} & =0,
\end{aligned}
$$

they are solved independently, as in section 2.2.1 giving the following results in the standard gauge

$$
\begin{aligned}
\left.X_{1}\right|_{\text {const. }} & =\xi_{1} \mathbb{1}_{2 \times 2}, \\
\left.Y_{1}\right|_{\text {const. }} & =\eta_{1} \mathbb{1}_{2 \times 2},
\end{aligned}
$$

where $\xi_{1}$ and $\eta_{1}$ are real constants, used as expansion parameters. In this gauge, we have the following identification of connections

$$
\left.R_{1 \mu}\right|_{\text {const. }}=\left.L_{1 \mu}\right|_{\text {const. }}=g_{1} G_{1 \mu} .
$$

\subsubsection{Bosons}

Going back to the unconstrained lagrangian, we find that the $\mathcal{O}\left(p^{2} \epsilon^{0}\right)$ lagrangian for the bosonic sector of this model is

$$
\begin{aligned}
\mathcal{L}_{\text {bosons }}^{(2,0)} & =\frac{f_{0}^{2}}{4}\left\langle D_{\mu} \Sigma_{0,1} D^{\mu} \Sigma_{0,1}^{\dagger}\right\rangle+\frac{f_{1}^{2}}{4}\left\langle D_{\mu} \Sigma_{1,2} D^{\mu} \Sigma_{1,2}^{\dagger}\right\rangle \\
& -\frac{1}{2}\left\langle G_{1 \mu \nu} G_{1}^{\mu \nu}\right\rangle-\frac{1}{2}\left\langle G_{0 \mu \nu} G_{0}^{\mu \nu}\right\rangle .
\end{aligned}
$$

Solving the constraints on the spurions, (5.63) becomes in the standard gauge used in section 5.2 .1

$$
\begin{aligned}
\left.\mathcal{L}_{\text {bosons }}^{(2,0)}\right|_{\text {const. }} & =\frac{f_{0}^{2}}{4}\left\langle\nabla_{\mu} \Sigma_{0,1} \nabla^{\mu} \Sigma_{0,1}^{\dagger}\right\rangle \\
& +\frac{f_{1}^{2}}{4}\left\langle\nabla_{\mu} \Sigma_{1,2} \nabla^{\mu} \Sigma_{1,2}^{\dagger}\right\rangle \\
& -\frac{1}{2}\left\langle G_{1 \mu \nu} G_{1}^{\mu \nu}\right\rangle-\frac{1}{4} b_{\mu \nu}^{0} b^{0 \mu \nu},
\end{aligned}
$$

where

$$
\begin{aligned}
\nabla_{\mu} \Sigma_{0,1} & =\left.D_{\mu} \Sigma_{0,1}\right|_{\text {const. }} \\
& =\partial_{\mu} \Sigma_{0,1}-\mathrm{i} g_{0} b_{\mu}^{0} \frac{\tau^{3}}{2} \Sigma_{0,1} \\
& +\mathrm{i} g_{1} \Sigma_{0,1} G_{1 \mu}, \\
\nabla_{\mu} \Sigma_{1,2} & =\left.D_{\mu} \Sigma_{1,2}\right|_{\text {const. }} \\
& =\partial_{\mu} \Sigma_{1,21}-\mathrm{i} g_{1} G_{1 \mu} \Sigma_{1,2} \\
& +\mathrm{i} g_{0} b_{\mu}^{0} \Sigma_{1,2} \frac{\tau^{3}}{2} .
\end{aligned}
$$

We also find plaquette terms ${ }^{18}$, which are the terms of lowest order in $\epsilon$ among those which carry no power of $p$. They are given by terms of the form

$$
\begin{gathered}
\left\langle\Sigma_{0,1} X_{1} Y_{1} \Sigma_{1,2} \tilde{X}_{0} \tilde{Y}_{0}\right\rangle \\
+\left\langle\tilde{Y}_{0}^{\dagger} \tilde{X}_{0}^{\dagger} \Sigma_{1,2}^{\dagger} Y_{1}^{\dagger} X_{1}^{\dagger} \Sigma_{0,1}^{\dagger}\right\rangle
\end{gathered}
$$

and similar ones when one replaces $\tilde{X}_{0}$ and $\tilde{Y}_{0}$ respectively by their conjugates $\tilde{X}_{0}^{c}$ and $\tilde{Y}_{0}^{c}$. There are four such terms, which give a tree-level mass-squared to the PGBs. We find therefore that the mass-squared of the PGBs is automatically counted as a small parameter of order $\mathcal{O}\left(p^{0} \epsilon^{4}\right)$.

We only briefly describe the field redefinitions as they are analogous to those of section 2.3 after injecting the solution to the constraints on spurions, we define the Goldstone boson fields remaining in the spectrum through

$$
\left.U\right|_{\text {const. }}=\Sigma_{0,1} \Sigma_{1,2}
$$

18 Note that one could in principle use the same spurion formalism to order the different possible plaquette terms in little Higgs models 62 according to an assumed pattern for the identification of symmetries. 
and the vector fields according to

$$
\begin{aligned}
\left.g_{1} W_{1 \mu}\right|_{\text {const. }} & =\mathrm{i} \Sigma_{0,1} \nabla_{\mu} \Sigma_{0,1}^{\dagger} \\
& -\left.\mathrm{i} \frac{\alpha_{1}}{g_{1}} U \nabla_{\mu} U^{\dagger}\right|_{\text {const. }},
\end{aligned}
$$

where

$$
\alpha_{1}=\frac{f_{1}^{2}}{f_{0}^{2}+f_{1}^{2}} .
$$

We then perform the change of variables

$$
\left\{G_{1 \mu}^{a}, b_{\mu}^{0}, \Sigma_{0,1}, \Sigma_{1,2}\right\} \longrightarrow\left\{W_{1 \mu}^{a}, b_{\mu}^{0}, U, \Sigma_{1,2}\right\},
$$

to find that $\Sigma_{1,2}$ does not appear anymore, due to the symmetries of the lagrangian. There remains one triplet of PGBs - collected in the unitary matrix $U$ - with decay constant $f_{\pi}$, which can be inferred from section 2.3 or 3.3.2 to be

$$
f_{\pi}^{2}=\frac{f_{0}^{2} f_{1}^{2}}{f_{0}^{2}+f_{1}^{2}}
$$

The mixing of $b_{\mu}^{0}$ and $W_{1 \mu}^{3}$ is identical to the case of section 4 with the replacement $f_{0}^{2} \longmapsto f_{0}^{2}+f_{1}^{2}$.

\subsubsection{Radiative corrections to the masses of PGBs}

The plaquette terms (5.67) do not contribute to the masssplitting within the PGB triplet. On the other hand, such a splitting arises from electroweak radiative corrections. A study of such corrections was first performed for the pions in the context of low-energy QCD in 37, and reformulated in the case of effective theories in [21 23. A consequence of the WSRs in this context is the softening of divergences in the one-loop corrections to the PGB masses ${ }^{19}$ : this is then another interpretation of the fact that the moose models yield softer corrections to PGB masses ${ }^{20}$, which has been the starting point of the little Higgs discussion 24, later generalized to a broader class of models that do not necessarily have a moose representation 65$]^{21}$. For simplicity we will consider the limit where the constant factors in front of the plaquette terms (5.67) appearing in the lagrangian are set to zero: the PGBs then have zero tree-level masses.

One may calculate the contribution of electroweak loops to the masses of the PGBs directly via Feynman diagrams.

\footnotetext{
19 In fact, in the SM, if weak corrections to the pion masses are considered in addition to the electromagnetic ones, the divergence is not quadratic, but only logarithmic even without the first Weinberg sum rule 63 .

20 See also 64 in this respect.

21 There are also connections with Randall-Sundrum 66] type models where EWSB is dictated by boundary conditions 60 67 61,68, as can be guessed from [69]: see footnote 17] In particular, the softness of corrections to two-point functions may well be one aspect of the general asymptotic softness property studied in 34 35.
}

However, we wish to explicitly show the implications of WSRs on this contribution and will therefore resort to the formulas given in 70, based on Dashen's results [71. This gives the correction to first order in $g_{0}^{2}$ as a convolution of the left-right two-point function studied in section [3] and of the correlator $\left\langle 0\left|T b_{\mu}^{0}(x) b_{\nu}^{0}(0)\right| 0\right\rangle$

$$
\begin{aligned}
& \left.\left(m_{\pi^{ \pm}}^{2}-m_{\pi^{0}}^{2}\right)\right|_{\text {loop }} \\
= & 4 \mathrm{i} \frac{g_{0}^{2}}{f_{\pi}^{2}} \int \mathrm{d}^{D} x\left\langle 0\left|T J_{L_{0}}^{3 \mu}(x) b_{\mu}^{0}(x) J_{R_{2}}^{3 \nu}(0) b_{\nu}^{0}(0)\right| 0\right\rangle \\
= & -\frac{g_{0}^{2}}{f_{\pi}^{2}} \int \mathrm{d}^{D} x\left\langle 0\left|T b_{\mu}^{0}(x) b_{\nu}^{0}(0)\right| 0\right\rangle \\
\times & \int \frac{\mathrm{d}^{D} q}{(2 \pi)^{D}} \mathrm{e}^{-\mathrm{i} q \cdot x}\left(\eta^{\mu \nu} q^{2}-q^{\mu} q^{\nu}\right) \Pi_{L R}\left(-q^{2}\right)
\end{aligned}
$$

However, $b_{\mu}^{0}$ is not a physical field, and we have to rewrite it in terms of $A_{\mu}$ and $Z_{\mu}$ : this is the reason why the formula is only the first term in an expansion. The effect of the full diagonalization is to replace the mass of the third component of the triplet $W_{1 \mu}^{a}$ by $M_{Z}^{2}$. When this is done, we are in a position to express the electroweak PGB mass shift as

$$
\begin{aligned}
& \left.\left(m_{\pi^{ \pm}}^{2}-m_{\pi^{0}}^{2}\right)\right|_{\text {loop }} \\
= & \mathrm{i} \frac{(D-1) e^{2}}{f_{\pi}^{2}} \int \frac{\mathrm{d}^{D} q}{(2 \pi)^{D}} \\
\times & \left(1+\left(\frac{s}{c}\right)^{2} \frac{q^{2}}{q^{2}-M_{Z}^{2}}\right) \Pi_{L R}\left(-q^{2}\right),
\end{aligned}
$$

with $\Pi_{L R}$ given by (3.22). The constants $e, c, s$ are defined as in section 4.2 This relation shows that the mass squared is only logarithmically divergent, and not quadratically divergent, due to the first Weinberg sum rule. This is true independently of the regularization method used. Note that, if we were to add at least another internal site to the chain, and thus one multiplet of resonances, we would get a convergent integral at this order. Finally, we find here

$$
\begin{aligned}
& \left.\left(m_{\pi^{ \pm}}^{2}-m_{\pi^{0}}^{2}\right)\right|_{\text {loop }} \\
= & -g_{0}^{2} M_{W}^{2}\left(6 \lambda+\frac{1}{16 \pi^{2}}\left(3 \ln \left(\frac{M_{Z}^{2}}{\mu^{2}}\right)+2\right)\right) \\
+ & \mathcal{O}(D-4)
\end{aligned}
$$

where the remaining logarithmic divergence is contained in the constant $\lambda$

$$
\lambda=\frac{\mu^{D-4}}{16 \pi^{2}}\left(\frac{1}{D-4}-\frac{1}{2}(\ln 4 \pi-\gamma+1)\right),
$$

and has to be canceled by the pole term in the appropriate counter-terms present at higher orders in the effective lagrangian. Such counter-terms will necessarily involve the spurions, and in fact, we note that we can build terms which encompass those of 22 when the constraints on the spurions are used: the following $\mathcal{O}\left(p^{0} \epsilon^{8}\right)$ term will absorb 
the divergences if our expansion allows it to appear at the same level as one-loop corrections

$$
\left\langle\tilde{Y}_{0}^{\dagger} \tilde{Y}_{0} \Sigma_{0,1} X_{1} Y_{1} \Sigma_{1,2} \tilde{X}_{0} \tilde{X}_{0}^{\dagger} \Sigma_{1,2}^{\dagger} Y_{1}^{\dagger} X_{1}^{\dagger} \Sigma_{0,1}^{\dagger}\right\rangle
$$

There are altogether sixteen such possible terms when one replaces the tilded spurions by their conjugates, though not all depend on the PGBs due to the relations

$$
\begin{gathered}
\tilde{X}_{0} \tilde{X}_{0}^{c \dagger}=\operatorname{det} X_{0} \mathbb{1}_{2 \times 2}, \\
\tilde{Y}_{0} \tilde{Y}_{0}^{c \dagger}=\operatorname{det} Y_{0} \mathbb{1}_{2 \times 2} .
\end{gathered}
$$

The fact that these counter-terms have to appear at oneloop to absorb the divergences to the masses of the PGBs then allows us to match the counting for the spurion expansion with that of the momentum expansion in this particular case where PGBs remain in the spectrum. We find that the correspondence between the two expansion parameters should then read, for our particular $K=1$ case

$$
\epsilon=\mathcal{O}\left(p^{1 / 2}\right)
$$

Note that, from the plaquette terms (5.67), this automatically gives in addition

$$
m_{\mathrm{PGBs}}^{2}=\mathcal{O}\left(p^{2}\right)
$$

and we already mention that, from the results in section 5.2.4 it would also imply for the fermions

$$
m_{\text {fermions }}=\mathcal{O}\left(p^{1}\right) \text {. }
$$

Both results are quite satisfactory from the point of view of reproducing the poles of the corresponding particles without resummation. Still, for the sake of generality, we shall refrain from using assumption (5.80) in the remainder of this paper.

\subsubsection{Fermions}

We now briefly describe the introduction of fermions in this model: the procedure is similar to that of section 4 . The model is summarized in Fig. 5.3 .

The elementary chiral fermions transform as

$$
\begin{aligned}
& \chi_{L} \longmapsto G_{1} \mathrm{e}^{-\mathrm{i} \frac{B-L}{2} \beta^{0}} \chi_{L}, \\
& \chi_{R} \longmapsto G_{0} \mathrm{e}^{-\mathrm{i} \frac{B-L}{2} \beta^{0}} \chi_{R},
\end{aligned}
$$

and the identification of $U$ (1) connections is performed as detailed in section 5.2.1 using the $\phi$ spurion. We find the following $\mathcal{O}\left(p^{2} \epsilon^{0}\right)$ lagrangian for fermions

$$
\begin{aligned}
\mathcal{L}_{\text {fermions }}^{(2,0)} & =\mathrm{i} \overline{\chi_{L}} \gamma^{\mu} D_{\mu} \chi_{L}+\mathrm{i} \overline{\chi_{R}} \gamma^{\mu} D_{\mu} \chi_{R} \\
& + \text { four-fermion interactions }
\end{aligned}
$$

giving the same leading-order couplings as in the SM.
The following $\mathcal{O}\left(p^{1} \epsilon^{2}\right)$ terms yield masses for the quarks

$$
\begin{aligned}
\mathcal{L}_{\text {quarks }}^{(1,2)} & =-m_{1 i j} \bar{\chi}_{L} X_{1}^{\dagger} \Sigma_{0,1}^{\dagger} \tilde{Y}_{0}^{\dagger} \chi_{R j} \\
& -m_{1 i j}^{*} \bar{\chi}_{j} \tilde{Y}_{0} \Sigma_{0,1} X_{1} \chi_{L i} \\
& -m_{2 i j} \bar{\chi}_{L} X_{1}^{\dagger} \Sigma_{0,1}^{\dagger} \tilde{Y}_{0}^{c \dagger} \chi_{R j} \\
& -m_{2 i j}^{*} \bar{\chi}_{j} \tilde{Y}_{0}^{c} \Sigma_{0,1} X_{1} \chi_{L i} \\
& -m_{3 i j} \bar{\chi}_{L} Y_{1} \Sigma_{1,2} \tilde{X}_{0} \chi_{R j} \\
& -m_{3 i j}^{*} \bar{\chi}_{j} \tilde{X}_{0}^{\dagger} \Sigma_{1,2}^{\dagger} Y_{1}^{\dagger} \chi_{L i} \\
& -m_{3 i j} \bar{\chi}_{L} Y_{1} \Sigma_{1,2} \tilde{X}_{0}^{c} \chi_{R j} \\
& -m_{4 i j}^{*} \bar{\chi}_{R} \tilde{X}_{0}^{c \dagger} \Sigma_{1,2}^{\dagger} Y_{1}^{\dagger} \chi_{L i},
\end{aligned}
$$

and there are again enough free parameters available per fermion doublet for the masses of the two states to be independent at tree-level. The additional parameters compared to the previous cases are not relevant for the masses and mixing, only for the Yukawa terms (see below). The usual term yielding Majorana masses for right-handed neutrinos are also present at this level. The power countings are as in section 4.5

Note in addition that, whatever the counting for $\epsilon$ is, we have the result that the masses of the fermions are counted at the same level as that of the PGBs

$$
m_{\text {fermions }}^{2}, m_{\mathrm{PGBs}}^{2}=\mathcal{O}\left(\epsilon^{4}\right) \text {. }
$$

On the other hand, the scalars remaining in the spectrum in this model bear little resemblance to the physical Higgs boson of the SM. In order to study this, one has to define the unitary gauge fermion fields through

$$
\begin{aligned}
\psi_{L} & =\Sigma_{0,1} U_{1} \chi_{L}, \\
\psi_{R} & =\chi_{R},
\end{aligned}
$$

yielding the same couplings of fermions to vector bosons as in section 4 These rewritings, together with those involving the vector bosons, yield a term

$$
\mathrm{i} \frac{f_{\pi}^{2}}{f_{0}^{2}} \overline{\psi_{L}} \gamma^{\mu}\left(U \nabla_{\mu} U^{\dagger}\right) \psi_{L} .
$$

which involves couplings of the fermions with the PGBs alone and with the PGBs and the neutral vector fields. The term linear in the PGB fields in (5.90) can be removed by a subsequent redefinition

$$
\begin{aligned}
& \Psi_{L}=\mathrm{e}^{-\mathrm{i} \frac{f_{\pi}}{f_{0}^{2}} \pi^{a} \tau^{a}} \psi_{L}, \\
& \Psi_{R}=\psi_{R},
\end{aligned}
$$

where

$$
U=\mathrm{e}^{\mathrm{i} \frac{\pi^{a} \tau^{a}}{f_{\pi}}}
$$

All the other terms involving interactions of the left-handed fermions with the PGBs are then modified, in particular the Yukawa terms. The fact that we have a triplet of PGBs and not a single boson - such as the physical 


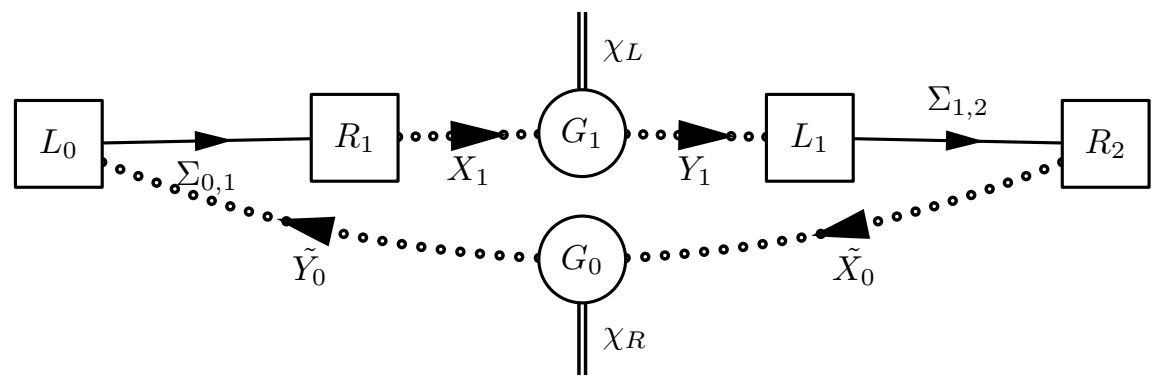

Fig. 5.3. Coupling fermions to the moose of section 5.2 .1

Higgs of the SM - and the remark following (5.86) on the number of terms imply that there is no simple relation between the masses and Yukawa couplings of fermions to the PGBs. Still, one would for instance expect general orderof-magnitude relations to hold: the Yukawa couplings of the third generation will be larger, except if particular cancellations occur.

We conclude this section by recalling the salient points encountered in the discussion of this model: we have presented the first approximation in the low-energy expansion for a model of EWSB where the only light particles not yet discovered at accelerators are a triplet of PGBs. We have discussed the way this model is derived from the moose idea, in the framework of effective theories, with emphasis on the spurion formalism. We have also described the consequences of the Weinberg sum rules derived in section 3 on the calculation of radiative corrections to the masses of the PGBs. We have pointed out that the terms we can build with our spurions do in particular allow us to renormalize the divergence in these masses: there is a connection with the study of Urech 22, provided we identified the $\epsilon$ power counting with the proper factors in momentum expansion (in fact with powers of gauge coupling constants). It is reassuring to find that this power counting in turn yields quite sensible results for the power counting of the masses of scalars and fermions. The three scalars remaining in the spectrum have Yukawa interactions with the fermions; however the connection between the coupling constants and the masses is not so direct as would be in the SM for instance.

\section{Conclusion}

In this paper, we have proposed to restore naturalness in Higgs-less EWSB effective theories within a systematic spurion formulation. The spurion formalism starts with a symmetry group $S_{\text {natural }}$, larger than the symmetry group $S_{\text {reduced acting on the low-energy degrees of free- }}$ dom. Both the restriction of the space of gauge connections and the coupling of Goldstone bosons to gauge fields follow from the condition of covariant constancy imposed on the spurions. This constraint effectively reduces the symmetry to $S_{\text {reduced }}$.

The spurions in fact play a double role. Their first use is to select the vacuum alignment of gauge fields $S_{\text {reduced }} \subset$
$S_{\text {natural }}$. This alignment is maintained in the limit of vanishing spurions. This effect of spurions is therefore already visible in the $\mathcal{O}\left(p^{2} \epsilon^{0}\right)$ part of the lagrangian although the latter does not explicitly involve any spurions. The second role of the spurions is to provide expansion parameters: when the constraints are solved in the standard gauge, the spurions reduce to constants, which we assume to be small.

To begin with a simpler example before focusing on EWSB, we have first used our spurion formalism in connection with a low-energy description of $K$ vector resonances coupled to the Goldstone bosons of a global chiral symmetry. The couplings at lowest order in the spurion expansion are identical to those obtained in the dimensional deconstruction approach. We therefore have an independent and more general bottom-up approach to justify this choice of leading-order interactions. We have shown that a set of $K$ generalized Weinberg sum-rules follow at treelevel. Corrections will only occur at higher orders in the spurion expansion.

Turning to EWSB, we have identified the set of spurions necessary to reduce the natural symmetry to the one that is gauged: $\mathrm{SU}(2) \times \mathrm{U}(1)_{Y}$. Known difficulties usually associated with Higgs-less theories at leading order are relegated to higher orders in the spurion expansion. This concerns $\mathcal{O}\left(p^{2}\right)$ contributions to the $S$ parameter and nonstandard couplings of the fermions to gauge fields (including non-universal couplings of the left-handed fermions and couplings of right-handed fermions to the $\left.W^{ \pm}\right)$. Another well-known difficulty of Higgs-less theories is to account for mass-splittings within fermion doublets at the same chiral order as the masses themselves. This finds a solution within the spurion formalism: as shown in section 4 the full CKM structure can be recovered. Also, due to the presence of the spurion $\phi$, whose leading couplings only appear in the neutrino sector, lepton number violation is introduced. One should stress the unifying aspect of the spurion approach, which offers a simultaneous solution to seemingly unrelated problems. Indeed, the scope of the formalism was further illustrated in section 5 with two extensions of the minimal effective theory of EWSB based on larger natural symmetries and therefore on a larger set of light states protected by them. These protected states involve either a tower of excited vector bosons or PGBs which remain in the spectrum. Such scalars are kept naturally light but share little resemblance with the SM Higgs boson. Examination of the spurion contributions to the 
tree-level as well as to the radiative masses of the PGBs suggests that the parameters $\xi$ and $\eta_{0}$ descending from the spurions might be of chiral order $\epsilon=\mathcal{O}\left(p^{1 / 2}\right)$. This in turn would imply that the leading-order contribution to the (Dirac) fermion masses arising from spurions be counted as $m_{\text {fermions }}=\mathcal{O}\left(p^{1}\right)$, as one would expect in a low-energy expansion.

On the other hand, higher orders in the spurion expansion certainly require a more complete analysis. First, the assumption of a common power counting, which we have been using for simplicity, is by no means granted. For instance, in the generic minimal EWSB effective theory of section [4 the spurion $\phi$ which allows for lepton number violation need not carry the same power counting as the real spurion $X_{1}$ responsible for the identification of two SU (2) groups or as the complex spurion $\tilde{Y}_{0}$ responsible for the selection of the $\mathrm{U}(1)_{\tau^{3}}$ group and for the introduction of isospin breaking. Furthermore, one needs to understand better how the spurion expansion fits in the whole low-energy expansion including loops. This requires a study of the way the powers of coupling constants and the constants descending from spurions feed back into each other in the renormalization procedure. A complete loop-level investigation going beyond the partial results of section 5.2 .3 would be in order.

Insight into the magnitude of the spurions relative to each other and relative to loops could be gained from the phenomenology of flavor violation, in particular in the lepton sector (using information from rare $K$ decays and from neutrino experiments for example). There obviously remains a lot of work to be done for the completion of this program. Needless to say, our formalism may also well be relevant outside the scope of Higgs-less EWSB.

Acknowledgements. We would like to thank Andreas Nyffeler for sharing his opinions and knowledge with us, and Bachir Moussallam for his interest and continuous encouragements. We also benefited from discussing some of the ideas exposed in this paper with Marc Knecht, Hagop Sazdjian and Mike Pennington.

This work was supported in part by the European Community EURIDICE network under contract HPRN-CT-200200311.

\section{A Resonance corrections to electroweak relations}

In this appendix, we give details about calculations for the oblique parameters in the model of section 5.1 evaluating the Fermi constant from muon decay, a contribution from $W^{\prime}$ exchange has to be taken into account, to find

$$
\begin{aligned}
G_{\mu} & =\frac{g_{2}^{2}}{4 \sqrt{2}}\left(\frac{\cos ^{2} \gamma}{M_{W}^{2}}+\frac{\sin ^{2} \gamma}{M_{W^{\prime}}^{2}}\right) \\
& =\frac{\sqrt{2}}{f_{1}^{2}} .
\end{aligned}
$$

The effective angle measured in low-energy $\nu N$ scattering is then

$$
\begin{aligned}
s_{*}^{2}(0) & =-\frac{g_{0}}{g_{2}}\left(\frac{\frac{N_{12} N_{22}}{M_{Z}^{2}}+\frac{N_{13} N_{23}}{M_{Z^{\prime}}^{2}}}{\frac{N_{22}^{2}}{M_{Z}^{2}}+\frac{N_{23}^{2}}{M_{Z^{\prime}}^{2}}}\right) \\
& =\frac{g_{0}^{2}}{g_{2}^{2}+g_{0}^{2}}+\frac{1}{2 g_{1}^{2}} \frac{g_{0}^{2} g_{2}^{2}\left(g_{2}^{2}-g_{0}^{2}\right)}{\left(g_{2}^{2}+g_{0}^{2}\right)^{2}} \\
& +\mathcal{O}\left(\frac{1}{g_{1}^{4}}\right) .
\end{aligned}
$$

Turning to the comparison between $G_{\mu}$ and the four-fermion terms generated by $Z$ and $Z^{\prime}$ exchange, one finds at treelevel

$$
\begin{aligned}
\rho_{*}(0) & =\frac{g_{2}^{2}}{4 \sqrt{2} G_{\mu}}\left(\frac{N_{22}^{2}}{M_{Z}^{2}}+\frac{N_{23}^{2}}{M_{Z^{\prime}}^{2}}\right) \\
& =1 .
\end{aligned}
$$

This can be understood as the consequence of the custodial symmetry, as noted in [72,46, in relation with 73 . However this symmetry, embodied here by SU $(2)_{L_{0}}$, does not enforce $T=0$, nor $\rho=1$, which are different in such cases [74. Indeed, to define $\rho$, we first have to define the angle appearing in the $Z^{0}$ couplings

$$
\begin{aligned}
s_{f}^{2} & =\frac{1}{4}\left(1-\frac{g_{V}}{g_{A}}\right) \\
& =-\frac{g_{0} N_{12}}{g_{2} N_{22}} \\
& =\frac{g_{0}^{2}}{g_{2}^{2}+g_{0}^{2}}+\frac{1}{2 g_{1}^{2}} \frac{g_{0}^{2} g_{2}^{2}\left(g_{2}^{2}-g_{0}^{2}\right)}{\left(g_{2}^{2}+g_{0}^{2}\right)^{2}} \\
& +\mathcal{O}\left(\frac{1}{g_{1}^{4}}\right),
\end{aligned}
$$

where $g_{V}$ and $g_{A}$ are the vector and axial couplings of charged leptons to the $Z^{0}$. We then find

$$
\begin{aligned}
\rho & =\frac{1}{1-s_{f}^{2}} \frac{M_{W}^{2}}{M_{Z}^{2}} \\
& =1-\frac{1}{4} \frac{g_{0}^{2}}{g_{1}^{2}}+\mathcal{O}\left(\frac{1}{g_{1}^{4}}\right),
\end{aligned}
$$

and we note that $s_{*}^{2}(0)$ and $s_{f}^{2}$ are different

$$
s_{*}^{2}(0)-s_{f}^{2}=\frac{1}{8} \frac{g_{0}^{2} g_{2}^{2}}{g_{1}^{4}} \frac{g_{0}^{2}-g_{2}^{2}}{g_{0}^{2}+g_{2}^{2}}+\mathcal{O}\left(\frac{1}{g_{1}^{6}}\right) .
$$

Turning now to the $S, T, U$ parameters, we get, from the definition

$$
\begin{aligned}
\Gamma\left(Z^{0} \longrightarrow l^{+} l^{-}\right) & =\frac{G_{\mu} M_{Z}^{3}}{24 \sqrt{2} \pi}\left(1-4 s_{f}^{2}+8 s_{f}^{4}\right) \\
& \times(1+\alpha T),
\end{aligned}
$$

the following negative contribution to $T$

$$
\begin{aligned}
\alpha T & =\frac{g_{2}^{2} N_{22}^{2}}{4 \sqrt{2} G_{\mu} M_{Z}^{2}}-1 \\
& =-\frac{1}{16 g_{1}^{4}}\left(g_{0}^{2}-g_{2}^{2}\right)^{2}+\mathcal{O}\left(\frac{1}{g_{1}^{6}}\right) .
\end{aligned}
$$


Defining the $S$ and $U$ parameters requires the introduction of $s_{0}^{2}$ through

$$
s_{0}^{2}\left(1-s_{0}^{2}\right)=\frac{\pi \alpha}{\sqrt{2} G_{\mu} M_{Z}^{2}},
$$

in order to extract $S$ from the definition

$$
s_{f}^{2}=s_{0}^{2}-\frac{s_{0}^{2}\left(1-s_{0}\right)^{2}}{1-2 s_{0}^{2}} \alpha T+\frac{1}{4\left(1-2 s_{0}^{2}\right)} \alpha S .
$$

The value of the $S$ parameter at tree-level has already been given in section 5.1 .2

Using the following definition for $U$

$$
\begin{aligned}
\frac{1}{1-s_{0}^{2}} \frac{M_{W}^{2}}{M_{Z}^{2}} & =1+\frac{1-s_{0}^{2}}{1-2 s_{0}^{2}} \alpha T-\frac{1}{2\left(1-2 s_{0}^{2}\right)} \alpha S \\
& +\frac{1}{4 s_{0}^{2}} \alpha U
\end{aligned}
$$

yields a positive value for $U$

$$
\alpha U=\frac{1}{4 g_{1}^{4}} g_{0}^{2} g_{2}^{2}+\mathcal{O}\left(\frac{1}{g_{1}^{6}}\right) .
$$

From all these expressions, one sees how the decoupling of the $W^{\prime}$ and $Z^{\prime}$ is achieved in the limit $g_{1} \longrightarrow+\infty$, in which one obtains a shorter moose - that of section 4

\section{References}

1. S. Weinberg, Physica A96, 327 (1979).

2. G. 't Hooft, in Recent developments in gauge theories, edited by G. 't Hooft et al., Plenum Press (1980).

3. J. Gasser and H. Leutwyler, Ann. Phys. 158, 142 (1984).

4. J. Gasser and H. Leutwyler, Nucl. Phys. B250, 465 (1985).

5. A. Dobado, D. Espriu, and M. J. Herrero, Phys. Lett. B255, 405 (1991).

6. D. Espriu and M. J. Herrero, Nucl. Phys. B373, 117 (1992).

7. The LEP Electroweak Working Group and the SLD Heavy Flavour Working Group (2002), hep-ex/0212036

8. M. E. Peskin and T. Takeuchi, Phys. Rev. D46, 381 (1992).

9. T. Appelquist and C. W. Bernard, Phys. Rev. D22, 200 (1980).

10. A. C. Longhitano, Phys. Rev. D22, 1166 (1980).

11. A. C. Longhitano, Nucl. Phys. B188, 118 (1981).

12. A. Nyffeler and A. Schenk, Phys. Rev. D53, 1494 (1996), hep-ph/9507287

13. H. Georgi, Nucl. Phys. B331, 311 (1990).

14. U.-G. Meißner, Phys. Rept. 161, 213 (1988).

15. G. Ecker, J. Gasser, H. Leutwyler, A. Pich, and E. de Rafael, Phys. Lett. B223, 425 (1989).

16. S. Weinberg, Phys. Rev. Lett. 18, 507 (1967).

17. D. T. Son and M. A. Stephanov (2003), hep-ph/0304182

18. H. Georgi, in Architecture of fundamental interactions at short distances, edited by P. Ramond, R. Stora, NorthHolland (1987).

19. N. Arkani-Hamed, A. G. Cohen, and H. Georgi, Phys. Rev. Lett. 86, 4757 (2001), hep-th/0104005
20. H.-C. Cheng, C. T. Hill, S. Pokorski, and J. Wang, Phys. Rev. D64, 065007 (2001), hep-th/0104179.

21. G. Ecker, J. Gasser, A. Pich, and E. de Rafael, Nucl. Phys. B321, 311 (1989).

22. R. Urech, Nucl. Phys. B433, 234 (1995), hep-ph/9405341

23. B. Moussallam, Nucl. Phys. B504, 381 (1997), hep-ph/9701400

24. N. Arkani-Hamed, A. G. Cohen, and H. Georgi, Phys. Lett. B513, 232 (2001), hep-ph/0105239

25. H. Leutwyler, Ann. Phys. 235, 165 (1994), hep-ph/9311274

26. J. Wess and B. Zumino, Phys. Lett. B37, 95 (1971).

27. J. Wudka, Int. J. Mod. Phys. A9, 2301 (1994), hep-ph/9406205

28. D. J. Gross and R. Jackiw, Phys. Rev. D6, 477 (1972).

29. C. Grosse-Knetter and R. Kögerler, Phys. Rev. D48, 2865 (1993), hep-ph/9212268

30. A. Manohar and H. Georgi, Nucl. Phys. B234, 189 (1984).

31. H. Georgi, Weak interactions and modern particle theory, Benjamin/Cummings (1984).

32. H. Georgi, Phys. Lett. B298, 187 (1993), hep-ph/9207278

33. S. Chang and H.-J. He (2003), hep-ph/0311177

34. R. Sekhar Chivukula, D. A. Dicus, and H.-J. He, Phys. Lett. B525, 175 (2002), hep-ph/0111016

35. C. Csáki, C. Grojean, H. Murayama, L. Pilo, and J. Terning (2003), hep-ph/0305237

36. M. A. Shifman, A. I. Vainshtein, and V. I. Zakharov, Nucl. Phys. B147, 385 (1979).

37. T. Das, G. S. Guralnik, V. S. Mathur, F. E. Low, and J. E. Young, Phys. Rev. Lett. 18, 759 (1967).

38. J. J. Sakurai, Currents and mesons, Univ. of Chicago Press (1969).

39. N. S. Craigie and J. Stern, Fortschr. Phys. 34, 261 (1986).

40. M. Knecht and E. de Rafael, Phys. Lett. B424, 335 (1998), hep-ph/9712457

41. E. Witten, Phys. Rev. Lett. 51, 2351 (1983).

42. G. 't Hooft, Nucl. Phys. B72, 461 (1974).

43. G. C. Rossi and G. Veneziano, Nucl. Phys. B123, 507 (1977).

44. E. Witten, Nucl. Phys. B160, 57 (1979).

45. L. Susskind, Phys. Rev. D20, 2619 (1979).

46. R. Sekhar Chivukula, H.-J. He, J. Howard, and E. H. Simmons (2003), hep-ph/0307209

47. S. Dimopoulos and L. Susskind, Nucl. Phys. B155, 237 (1979).

48. T. Appelquist, M. J. Bowick, E. Cohler, and A. I. Hauser, Phys. Rev. D31, 1676 (1985).

49. R. D. Peccei and X. Zhang, Nucl. Phys. B337, 269 (1990).

50. R. Casalbuoni, S. De Curtis, D. Dominici, and R. Gatto, Nucl. Phys. B282, 235 (1987).

51. R. Casalbuoni, S. De Curtis, D. Dominici, F. Feruglio, and R. Gatto, Int. J. Mod. Phys. A4, 1065 (1989).

52. M. Knecht and R. Urech, Nucl. Phys. B519, 329 (1998), hep-ph/9709348

53. E. Bagan, D. Espriu, and J. Manzano, Phys. Rev. D60, 114035 (1999), hep-ph/9809237

54. A. Nyffeler and A. Schenk, Phys. Rev. D62, 113006 (2000), hep-ph/9907294

55. P. Sikivie, L. Susskind, M. B. Voloshin, and V. I. Zakharov, Nucl. Phys. B173, 189 (1980).

56. R. Casalbuoni et al., Phys. Lett. B349, 533 (1995), hep-ph/9502247 
57. M. Bando, T. Kugo, and K. Yamawaki, Phys. Rept. 164, 217 (1988).

58. G. Altarelli and R. Barbieri, Phys. Lett. B253, 161 (1991).

59. G. Sánchez-Colón and J. Wudka, Phys. Lett. B432, 383 (1998), hep-ph/9805366

60. C. Csáki, C. Grojean, L. Pilo, and J. Terning (2003), hep-ph/0308038

61. Y. Nomura, JHEP 11, 050 (2003), hep-ph/0309189

62. N. Arkani-Hamed et al., JHEP 0208, 021 (2002), hep-ph/0206020

63. M. Knecht, S. Peris, and E. de Rafael, Phys. Lett. B443, 255 (1998), hep-ph/9809594

64. K. Lane, Phys. Rev. D65, 115001 (2002), hep-ph/0202093

65. N. Arkani-Hamed, A. G. Cohen, E. Katz, and A. E. Nelson, JHEP 0207, 034 (2002), hep-ph/0206021

66. L. Randall and R. Sundrum, Phys. Rev. Lett. 83, 3370 (1999), hep-ph/9905221

67. R. Contino, Y. Nomura, and A. Pomarol (2003), hep-ph/0306259

68. H. Davoudiasl, J. L. Hewett, B. Lillie, and T. G. Rizzo (2003), hep-ph/0312193

69. R. Barbieri, A. Pomarol, and R. Rattazzi (2003), hep-ph/0310285

70. M. E. Peskin, Nucl. Phys. B175, 197 (1980).

71. R. F. Dashen, Phys. Rev. D3, 1879 (1971).

72. R. Casalbuoni et al., Phys. Rev. D53, 5201 (1996), hep-ph/9510431

73. H. Georgi and S. Weinberg, Phys. Rev. D17, 275 (1978).

74. S. Chang and J. G. Wacker (2003), hep-ph/0303001 